\title{
High Dietary Fat Consumption Impairs Axonal Mitochondrial Function In Vivo
}

\author{
Marija Sajic, ${ }^{1 *}$ Amy E. Rumora, ${ }^{2 *}$ Anish A. Kanhai, ${ }^{1}$ Giacomo Dentoni, ${ }^{1}$ Sharlini Varatharajah, ${ }^{1}$ Caroline Casey, ${ }^{1}$ \\ ${ }^{1}$ Ryan D. R. Brown, ${ }^{1}$ Fabian Peters, ${ }^{1}$ Lucy M. Hinder, ${ }^{2}$ Masha G. Savelieff, ${ }^{3}{ }^{\circledR}$ Eva L. Feldman, ${ }^{2}$ and \\ ${ }^{\circ}$ Kenneth J. Smith ${ }^{1}$ \\ ${ }^{1}$ Department of Neuroinflammation, UCL Queen Square Institute of Neurology, London, WC1N 3BG, United Kingdom, ${ }^{2}$ Department of Neurology, \\ University of Michigan, Ann Arbor, Michigan 48109, and ${ }^{3}$ NeuroNetwork for Emerging Therapies, University of Michigan, Ann Arbor, Michigan 48109
}

Peripheral neuropathy (PN) is the most common complication of prediabetes and diabetes. PN causes severe morbidity for Type 2 diabetes (T2D) and prediabetes patients, including limb pain followed by numbness resulting from peripheral nerve damage. PN in T2D and prediabetes is associated with dyslipidemia and elevated circulating lipids; however, the molecular mechanisms underlying PN development in prediabetes and T2D are unknown. Peripheral nerve sensory neurons rely on axonal mitochondria to provide energy for nerve impulse conduction under homeostatic conditions. Models of dyslipidemia in vitro demonstrate mitochondrial dysfunction in sensory neurons exposed to elevated levels of exogenous fatty acids. Herein, we evaluated the effect of dyslipidemia on mitochondrial function and dynamics in sensory axons of the saphenous nerve of a male high-fat diet (HFD)-fed murine model of prediabetes to identify mitochondrial alterations that correlate with PN pathogenesis in vivo. We found that the HFD decreased mitochondrial membrane potential (MMP) in axonal mitochondria and reduced the ability of sensory neurons to conduct at physiological frequencies. Unlike mitochondria in control axons, which dissipated their MMP in response to increased impulse frequency (from 1 to $50 \mathrm{~Hz}$ ), HFD mitochondria dissipated less MMP in response to axonal energy demand, suggesting a lack of reserve capacity. The HFD also decreased sensory axonal $\mathrm{Ca}^{2+}$ levels and increased mitochondrial lengthening and expression of PGC1 $\alpha$, a master regulator of mitochondrial biogenesis. Together, these results suggest that mitochondrial dysfunction underlies an imbalance of axonal energy and $\mathrm{Ca}^{2+}$ levels and impairs impulse conduction within the saphenous nerve in prediabetic PN.

Key words: dorsal root ganglion; high-fat diet; mitochondria; mouse model; peripheral neuropathy; sensory neuron

\section{Significance Statement}

Diabetes and prediabetes are leading causes of peripheral neuropathy (PN) worldwide. PN has no cure, but development in diabetes and prediabetes is associated with dyslipidemia, including elevated levels of saturated fatty acids. Saturated fatty acids impair mitochondrial dynamics and function in cultured neurons, indicating a role for mitochondrial dysfunction in PN progression; however, the effect of elevated circulating fatty acids on the peripheral nervous system in vivo is unknown. In this study, we identify early pathogenic events in sensory nerve axons of mice with high-fat diet-induced PN, including alterations in mitochondrial function, axonal conduction, and intra-axonal calcium, that provide important insight into potential PN mechanisms associated with prediabetes and dyslipidemia in vivo.

Received July 15, 2020; revised Feb. 11, 2021; accepted Mar. 15, 2021.

Author contributions: M.S., A.E.R., L.M.H., E.L.F., and KJ.S. designed research; M.S., A.A.K., G.D., S.V., C.C., R.D.R.B., and F.P. performed research; M.S., A.E.R., F.P., A.A.K., S.V., C.C., and R.D.R.B. analyzed data; M.S., A.E.R., M.G.S., E.L.F., and K.J.S. wrote the first draft of the paper; M.S., A.E.R., A.A.K., G.D., S.V., C.C., R.D.R.B., F.P., L.M.H., M.G.S., E.L.F., and K.J.S. edited the paper.

This work was supported by the Leducq Foundation to M.S. and K.J.S.; National Institutes of Health 1T32DK101357, F32DK112642, and 1K99DK119366 to A.E.R.; National Institutes of Health R21NS102924 and R24DK082841 to E.L.F.; Novo Nordisk Foundation NNF140C0011633 to E.L.F.; and NeuroNetwork for Emerging Therapies. The complex lipid analysis was completed by the University of Cincinnati Medical Center MMPC supported by the U24 DK059630 Grant. We thank Dr. Oliver Griesbeck (Max Planck Institute) for the Tn-XXL mice; Dr. Stacey Sakowski Jacoby (University of Michigan) for expert editorial assistance; Faye E. Mendelson (University of Michigan) for technical assistance; and John Hayes (University of Michigan) for assistance with data visualization.

${ }^{*}$ M.S. and A.E.R. contributed equally to this work.

The authors declare no competing financial interests.

Correspondence should be addressed to Marija Sajic at m.sajic@ucl.ac.uk.

https://doi.org/10.1523/JNEUROSCI.1852-20.2021

Copyright $\odot 2021$ the authors

\section{Introduction}

Diabetes and prediabetes incidence is rising at alarming rates. In 2019, 463 million people globally had diabetes, with a projected increase to $\sim 700$ million by 2045 (Saeedi et al., 2019). Peripheral neuropathy $(\mathrm{PN})$, the most prevalent prediabetic and diabetic complication, affects $\sim 50 \%$ of Type 2 diabetes (T2D) patients and $33 \%$ of prediabetic patients in the United States (Feldman et al., 2019). Characterized by nerve damage, PN manifests as pain or sensory loss starting in the feet and then the hands in a "stocking and glove" pattern. At end-stages, complete loss of sensation in peripheral tissues results in morbidity, including gait problems, foot ulcerations, and lower limb amputation. Early attempts to prevent $\mathrm{PN}$ in prediabetes and T2D patients focused on glycemic control alone, which was only marginally successful 
(Callaghan et al., 2012). As the increased prevalence of prediabetes and T2D is associated with obesity and high-fat "Western" diets, saturated fatty acids (SFAs) and dyslipidemia likely play important roles in PN progression (Eid et al., 2019). Indeed, recent clinical studies have correlated PN progression with the metabolic syndrome and dyslipidemia in both prediabetes and T2D, independent of glycemic control (Callaghan et al., 2016a,b, 2018, 2020).

Although overwhelming preclinical and clinical evidence indicates that an SFA-rich diet correlates with PN development (Savelieff et al., 2020), molecular mechanisms remain poorly understood. DRG neurons, the primary sensory neurons affected by PN, have axons up to a $1 \mathrm{~m}$ long in humans and thus require long-distance axonal transport of functional mitochondria to satisfy distal ATP demands and maintain adequate $\mathrm{Ca}^{2+}$ buffering (Sajic, 2014). Elevated long-chain SFA levels impair mitochondrial metabolism (Schonfeld and Wojtczak, 2016), and we demonstrated that exogenous long-chain SFAs reduce mitochondrial membrane potential (MMP), bioenergetics capacity, ATP production, and motility of axonal mitochondria in cultured DRG neurons, while physiological glucose had no effect (Rumora et al., 2018, 2019a). Since PN is characterized by distal-to-proximal axonal degeneration, we hypothesized that SFA-induced impairment in mitochondrial function and transport in vivo is an important cause of $\mathrm{PN}$ progression.

Mitochondrial function has been investigated in vitro (Feldman et al., 2019), and in in vivo models where myelination affects mitochondrial function and dynamics (Zhang et al., 2010; Ohno et al., 2011; Sajic et al., 2013). In vitro, MMP and mitochondrial motility are constantly influenced by growth cues from neurite outgrowth in culture (Morris and Hollenbeck, 1993; Chada and Hollenbeck, 2004), but these cues are missing in vivo in healthy adult animals. Cultured or ex vivo preparations also lack a perfused vasculature to ensure physiological nutrient and oxygenation levels (Sajic, 2014). To overcome these constraints and validate our in vitro findings (Rumora et al., 2018, 2019a), we examined mitochondria in vivo within the homeostatically regulated environment of adult, electrically active saphenous nerve axons in a high-fat diet (HFD)-induced prediabetes mouse model. The HFD chow contains $45 \%$ lard, mimicking a Western SFA-rich diet (O'Brien et al., 2014). HFD-fed mice recapitulate salient features of prediabetes, including impaired glucose tolerance, obesity, and neuropathy, and they develop electrophysiological and histologic characteristics of human PN, for example, reduced sensory and motor nerve conduction velocities and decreased intraepidermal nerve fiber density (IENFD) (O'Brien et al., 2014), making it a useful model for evaluating PN mechanisms.

We report that mitochondrial transport in saphenous nerve axons of prediabetic HFD mice is intact in vivo. However, axonal mitochondria suffer from significantly reduced MMP, which does not dissipate in response to physiological increases in axonal energy demand in HFD mice versus control mice. These changes also associate with significant decreases in intra-axonal calcium levels $\left(\left[\mathrm{Ca}^{2+}\right]_{\mathrm{i}}\right)$ and lower expression of $\mathrm{PGCl} \alpha$, a master regulator mitochondrial biogenesis, in prediabetic versus control mice. These results suggest that partial MMP loss and reduced $\left[\mathrm{Ca}^{2+}\right]_{\mathrm{i}}$ play important roles in regulating sensory neuron axonal impulse conduction in peripheral nerves of prediabetic HFD mice with PN.

\section{Materials and Methods}

Animals

All experiments were conducted under the United Kingdom 1986 Animals (Scientific Procedures) Act, the United Kingdom 2006 Animal Welfare Act, and the European Directive 2010/63/EU. All experiments were approved by the institutional ethics committee and followed the ARRIVE guidelines. Mouse strains used in this study included positive and negative littermates of genetically modified Tn-XXL mice (Mank et al., 2008) and B6.CG-Tg(Thy1-CFP/COX8A)S2Lich/J mice (The Jackson Laboratory, catalog \#007967). All mice were housed in individually ventilated cages with a maximum of 5 mice per cage that were provided with bedding, nesting material, playing tunnels, and wood blocks. Mice had access to food, as specified in the experimental design, and water ad libitum, and were kept on a $12 \mathrm{~h}$ light/12 h dark cycle. At study termination, plasma was collected and submitted to the Cincinnati Mouse Metabolic Phenotyping Center for a complex lipid panel analysis to measure circulating phospholipids, cholesterol, triglycerides, and nonesterified fatty acids. Both male and female mice were also evaluated for sex differences related to food consumption, weight gain, hyperglycemia, glucose tolerance, and tactile hypersensitivity before assessment of in vivo mitochondrial events. Food consumption was evaluated by measuring the gram amount of food consumed per mouse per day for both male and female mice.

\section{Fasting glucose}

Blood glucose measurements were performed after overnight fasting, using a commercially available Accu-Check Mobile blood glucose monitor (Roche), from a single, minimally invasive tail puncture.

\section{Intraperitoneal glucose tolerance test (ipGTT)}

The ipGTT was performed according to the DiaComp protocol (https:// www.diacomp.org/shared/document.aspx?id=11\&docType=Protocol). Briefly, after the fasting period, mice were weighed, and a baseline blood glucose measurement was obtained, as described above. An intraperitoneal injection of glucose ( $1 \mathrm{mg} / \mathrm{g}$ ) (Sigma Millipore) was administered, and further blood glucose measurements were acquired 15, 30, 60, and $120 \mathrm{~min}$ after the intraperitoneal injection.

\section{Tactile hypersensitivity}

Tactile responses were evaluated as described previously (Obrosova et al., 2007). In short, mice were transferred into a clear Plexiglas box with mesh floor, and allowed to acclimatize. Following the acclimatization period, a series of flexible von Frey filaments (NC12775-99, North Coast) were applied to the surface of the hind paws with adequate force to bend the filament for $6 \mathrm{~s}$. Paws were stimulated alternately, and stimulations were repeated 6 times. Short paw withdrawal or paw flinching was classified as a positive response. Stimulation was stopped when $>50 \%$ of the 6 stimuli were positive; otherwise, the filament with the next greater force was applied. Tactile response thresholds were calculated with the formula $\left[\left(\mathrm{w}_{1} \times \mathrm{r}_{1}\right)+\left(\mathrm{w}_{2} \times \mathrm{r}_{2}\right)+\left(\mathrm{w}_{\mathrm{x}} \times \mathrm{r}_{\mathrm{x}}\right) / \mathrm{r}_{\text {total }}\right]$, with $\mathrm{w}$ corresponding to filament weight and $r$ corresponding to number of positive responses for that filament.

\section{Experimental design}

Mitochondrial dynamics experiments. Cyan fluorescent protein (CFP) transgenic mice (B6.Cg-Tg(Thy1-CFP/COX8A)S2Lich/J, The Jackson Laboratory, catalog \#007967) with constitutive expression in $40 \%-60 \%$ of mitochondria were used for imaging mitochondria in vivo. Male littermates were randomly assigned to either a control chow diet (Lab Diet 5001 catalog \#0001319, 13\% kcal fat) or HFD (Research Diets catalog \#D12451, 45\% kcal fat) according to the following regimens: (1) 4 -week-old mice fed control or HFD for 16 weeks $(4+16$ group, aged 20 weeks at experiment termination), (2) 4-week-old mice fed control or HFD for 36 weeks ( $4+36$ group, aged 40 weeks at experiment termination), or (3) 24-week-old mice fed control or HFD for 16 weeks $(24+16$, aged 40 weeks at experiment termination). Dietary composition, fatty acid makeup, and energy density of the control diet and HFD chows are detailed in Extended Data Fig. 1-1. Animals were weighed weekly and examined for signs of pain or discomfort.

Calcium imaging experiments. Tn-XXL transgenic mice, which express a calcium-binding troponin C-based biosensor with two fluorescent probes, citrine and CFP, were used for imaging calcium (Mank et al., 2008). Two experiments were performed. In the first, 9-week-old male littermates were randomly assigned to either a control diet or HFD 
for 5 weeks ( $9+5$ group, aged 14 weeks at experiment termination). In the second, 24-week-old male littermates were randomly assigned to either a control diet or HFD for 16 weeks $(24+16$ group, aged 40 weeks at experiment termination).

\section{Surgical procedure, in vivo imaging, and electrophysiology}

Animals were terminally anesthetized (urethane, $1.25 \mathrm{~g} / \mathrm{kg}$, i.p.) (Sigma Millipore), the leg was immobilized using a mold made from dental polymer (Examix NDS Monophase Purple, GC America). The left saphenous nerve was exposed at three locations: at the mid-thigh for confocal imaging, at the groin for the stimulating electrode, and at the ankle for the recording electrode. The left saphenous nerve exposed at midthigh was desheathed, as described previously (Sajic et al., 2013), and stained in situ with tetramethylrhodamine methyl ester [TMRM (Invitrogen/Thermo Fisher Scientific); $0.5 \mu \mathrm{m}$ in aCSF (Harvard Apparatus) for $30 \mathrm{~min}$ ], and the surrounding structures with fluorescein isothiocyanate-conjugated Griffonia isolectin B4 (1:20 in sterile saline for $10 \mathrm{~min}$ ) (Sigma Millipore), in addition to the constitutive CFP labeling. While the dyes incubated at the mid-thigh, the saphenous nerve was exposed in the groin to create a "well" with the exposed part of the nerve at its bottom. Teflon tape, $\sim 2 \mathrm{~cm} \times 0.5 \mathrm{~cm}$, was placed under the nerve to electrically isolate it from the surrounding muscle. A pair of stimulating platinum wire electrodes was placed under the saphenous nerve, resting on the Teflon tape. The well was then filled with mineral oil to prevent tissue drying. Next, the saphenous nerve was exposed at the ankle to attach the active recording electrode, which was placed directly next to the exposed distal part of the saphenous nerve. A reference needle electrode was inserted into the fifth toe, and a ground electrode was placed in the abdominal muscle. At this stage, the animal was transferred to the confocal microscope (LSM Pascal 710, Carl Zeiss). Fresh saline was applied to the exposed saphenous nerve at mid-thigh and covered with a glass coverslip sealed with petroleum jelly. Confocal time-lapse and single images were taken using either a Zeiss Apochromat $10 \times$ objective (NA 0.3; overview only) or a Zeiss Apochromat Plan $63 \times$ oil objective (NA 1.4). The pinhole size was set to $1 \mathrm{AU}$, and time-lapse sequences were recorded at 1 frame/s for $100 \mathrm{~s}$. Successive dual-color CFP/TMRM $(405 \mathrm{~nm} /$ $543 \mathrm{~nm}$ ) images were taken to distinguish axonal mitochondria from mitochondria found in other cells. However, time-lapses were based on the TMRM signal alone because of its superior contrast and because repeated CFP imaging causes a prohibitive amount of bleaching. Time-lapse images of mitochondria were taken simultaneously with electrophysiological stimulation and recording. First, a single supramaximal stimulus was delivered at frequencies of 1 and $50 \mathrm{~Hz}$. Sensory compound action potentials (sCAPs) were averaged $(n=30)$ and stored (Sigma 60, Nicolette Technologies). Next, to examine the refractory period of transmission (RPT), two successive supramaximal stimuli were delivered while sequentially increasing the delay between the first and second stimulus in $0.1 \mathrm{~ms}$ increments, up to $2.5 \mathrm{~ms}$. sCAPs were averaged $(n=30)$ and stored (Sigma 60, Nicolette Technologies). The amplitudes were measured using MATLAB.

\section{In vivo calcium imaging}

Tn-XXL mice were prepared for imaging as described above. However, no dyes were applied to the nerve, and images were acquired using a Zeiss Apochromat Plan $63 \times$ oil objective (NA 1.4). First, axons of interest were identified by exciting the citrine fluorophore of Tn-XXL directly via a $514 \mathrm{~nm}$ laser. Next, two $512 \times 512$ pixel images were taken for every axon: (1) a "ratiometric" two-channel image with an excitation of $405 \mathrm{~nm}$ and detection ranges of 460-510 nm (for CFP) and 520-570 nm (for citrine); and (2) an "anatomic" image with $514 \mathrm{~nm}$ excitation and a broad detection range of 515-650 $\mathrm{nm}$. Laser settings were constant across all animals. The initial calibration was performed in naive Tn-XXL animals, which was removed from the microscope stage after visible axons were imaged. Then ionomycin (100 $\mu \mathrm{M}$ in aCSF) was applied to the nerve for $10 \mathrm{~min}$, a new coverslip was placed over the leg, and imaging was repeated.

\section{Tissue collection}

After sCAPs and/or images were acquired, mice were perfused transcardially with PFA (4\% in $0.4 \mathrm{M} \mathrm{PB}, \mathrm{pH} 7.4$; VWR Chemicals). Sciatic nerves and spinal cord lumbar segments (L3, L4, and L5) were dissected and postfixed for $2 \mathrm{~h}$ (PFA, $4 \%$ in $0.4 \mathrm{M} \mathrm{PB}$ ), whereas glabrous and hairy skin were postfixed overnight. After postfixation, tissues were transferred to $0.1 \%$ sodium azide (VWR Chemicals) in PBS. A block of hairy skin from the medial side of the foot dorsum, innervated by saphenous nerve, and a block of glabrous skin from the foot plantar innervated by the sciatic nerve were dissected. Skin blocks and spinal cord tissue were separately embedded into paraffin wax. Eight micron sections were cut using a microtome (Leitz 1512), transferred into a water bath (Medlite Tissue Flotation Bath, TFB- 45, Medizintechnik) set at a temperature of $40^{\circ} \mathrm{C}$, mounted onto slides (Superfrost, Thermo Scientific), and left to air-dry overnight.

\section{Immunohistochemistry}

To assess PGC1 $\alpha$ expression in DRGs or skin IENFD, sections were incubated with anti-PGC1 $\alpha$ (Abcam, ab54481, RRID:AB_881987) and anti- $\beta$-III-tubulin (Abcam, ab78078, RRID:AB_2256751) antibodies, respectively. First, the slides were deparaffinized by incubating in xylene (VWR Chemicals) for $5 \mathrm{~min}$, xylene/100\% ethanol 1:1 for $3 \mathrm{~min}$, and then 3 min each in serial ethanol dilutions: $100 \%, 90 \%, 70 \%, 50 \%$ (VWR Chemicals). Slides were rinsed in distilled water for $3 \mathrm{~min}$ followed by cold acetone (VWR Chemicals) for $5 \mathrm{~min}$. After deparaffinization, the slides were washed twice in PBS for $5 \mathrm{~min}$. Antigen retrieval was performed by heating slides to boiling in $10 \%$ citrate solution ( $\mathrm{pH}$ 6; Dako) and then transferring to an oven at $40^{\circ} \mathrm{C}$ for $40 \mathrm{~min}$. The slides were then rinsed in distilled water, followed by two washes in PBS-T (PBS with $0.2 \%$ Triton X-100, Sigma Millipore). Slides were then blocked in $5 \%$ rabbit serum in PBS-T (Vector Laboratories) for $1 \mathrm{~h}$ in a humidified chamber at room temperature. The serum was removed with a Pasteur pipette, and the primary antibodies, polyclonal rabbit anti-PGC1 $\alpha$ (1:600) and monoclonal mouse anti-neuronal $\beta$-III tubulin $(1: 1000)$ diluted in $2 \%$ blocking solution were applied overnight at $4^{\circ} \mathrm{C}$. The next day, the slides were washed twice in PBS and once in distilled water, each for $5 \mathrm{~min}$. The goat anti-rabbit AlexaFluor-546 (Invitrogen Thermo Fisher Scientific, catalog \#A11010, RRID:AB_2534077) or goat antimouse AlexaFluor-647 (Invitrogen Thermo Fisher Scientific, catalog \#32728, RRID:AB_2633277) was added to their respective primary for 1 $\mathrm{h}$ in the dark. Next, the slides were washed 3 times in PBS for $5 \mathrm{~min}$ and then rinsed with distilled water. The slides were air dried in the dark. Coverslips were mounted using DAPI fluoromount (Vectashield, Vector Laboratories) and sealed using nail varnish. Additionally, in processing tissue for DRGs, a 70\% methanol (VWR Chemicals) pretreatment step was conducted after deparaffinization. Also, two rounds of incubation in $1 \%$ sodium borohydrite (Sigma-Merck) for 5 min each were conducted after antigen retrieval, followed by incubation in blocking solution with $20 \%$ rabbit serum. All sections were immunolabeled in the same session. For image acquisition, fluorescence emission was recorded through a $10 \times / 0.3$, Carl Zeiss EC Plan Neofluar $10 \times / 0.3 \mathrm{Ph} 2$ or Plan-Apochromat $63 \times / 1.4$ Oil Ph3 (Carl Zeiss) objective lens. All images were acquired with the following settings: beam-splitter HFT 488/543; filter BP 505570, argon laser irradiation at $488 \mathrm{~nm}$ and filter LP 585 helium neon laser irradiation at $543 \mathrm{~nm}$. First, tile scans were conducted using Zeiss EC Plan Neofluar $10 \times / 0.3 \mathrm{Ph} 2$ magnification using blue (DAPI, $405 \mathrm{~nm}$ ) and red (AlexaFluor-546, $565 \mathrm{~nm}$ ) channels. For images of $\beta$-III-tubulin-labeled hairy and glabrous skin sections, $Z$ stack images from five, randomly chosen, nonoverlapping positions were acquired using Zeiss EC Plan Neofluar $40 \times / 0.75$ Ph2 (water) objective (Carl Zeiss) and ZEN black acquisition software (Carl Zeiss). If the image did not appear to be uniformly labeled across the FOV, the next random area was chosen for image acquisition. Each $Z$ stack contained 10 images, each at $1 \mu \mathrm{m}$ thickness. For images of PGC1 $\alpha$-labeled DRGs, $Z$ stacks were taken in the same way, while using the Zeiss PlanApochromat $65 \times / 1.40$ (oil) $\mathrm{Ph} 3$ objective. Care was taken not to saturate pixel intensity during image acquisition. Tissue dissection, embedding, sectioning, immunolabeling, and image acquisition and image analysis were performed in a blinded fashion. 
TERMINAL confocal imaging of mitochondria

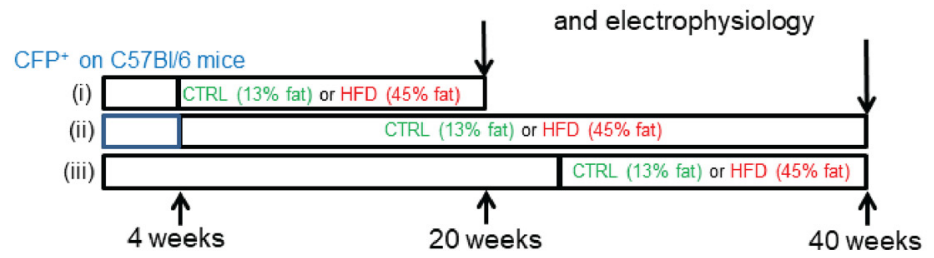

WEIGHT
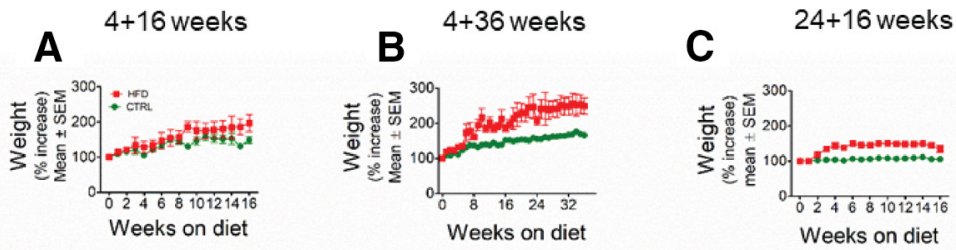

TERMINALFASTING GLUCOSE
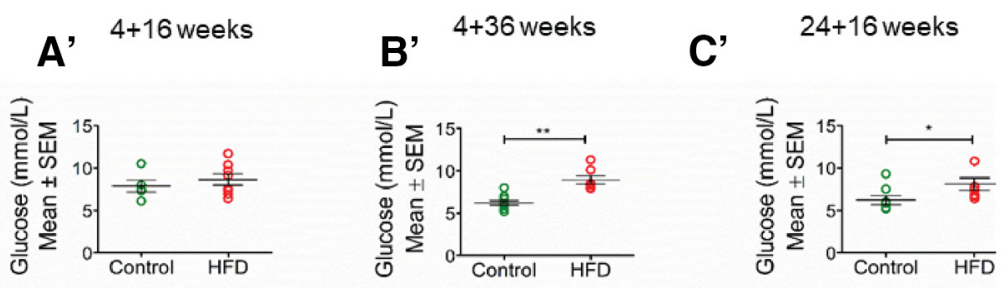

TERMINALIPGTT
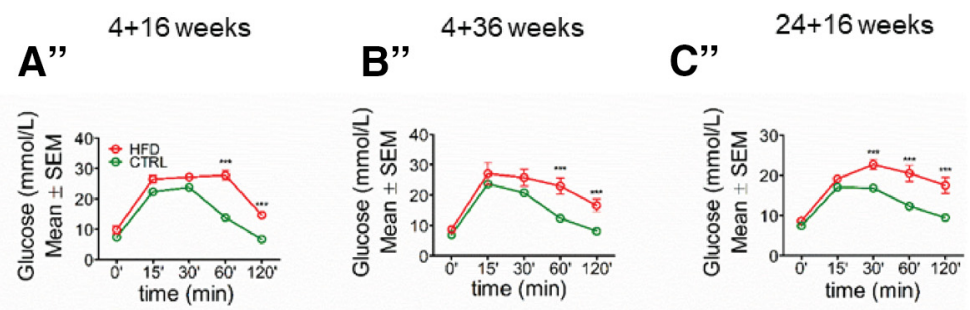

PLASMA LIPID LEVELS
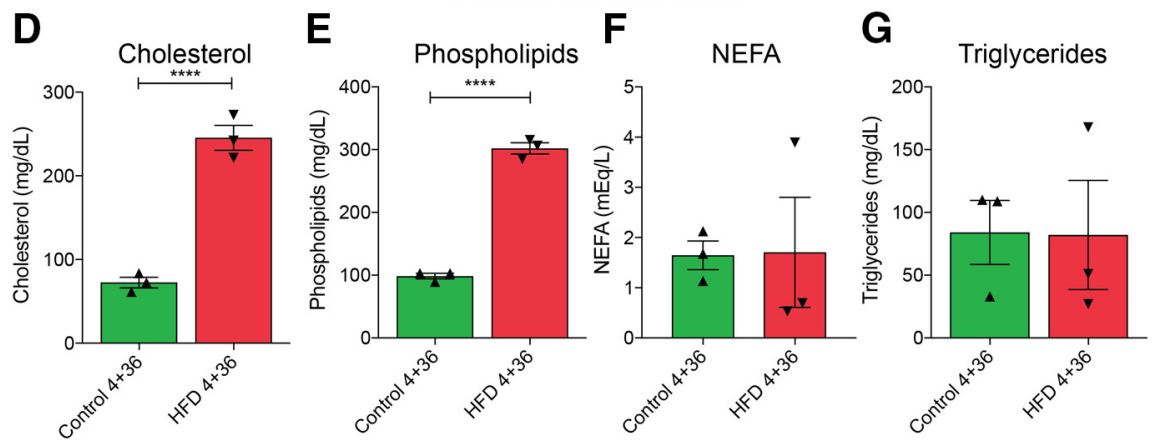

Figure 1. HFD mice exhibit weight gain and impaired glucose tolerance typical of prediabetes. Top, Scheme of the three experimental groups: (i) mice fed HFD from 4 weeks of age for 16 weeks $(4+16$ weeks); (ii) mice fed HFD from 4 weeks of age for 36 weeks ( $4+36$ weeks); and (iii) mice fed HFD from 24 weeks of age for 16 weeks $(24+16$ weeks). The control diet and HFD fatty acid composition are provided in Extended Data Figure 1-1. Respective control mice received control chow. At the endpoint, mitochondrial transport and morphology, and axonal $\left[\mathrm{Ca}^{2+}\right]_{\mathrm{j}}$, were measured in vivo along with electrophysiological measurements. $\boldsymbol{A}, \boldsymbol{B}, \mathrm{HFD} 4+16$ and $4+36$ mice gain more weight than their respective controls, and the percentage weight increase is significant starting from 15 and 19 weeks of HFD, respectively, and onwards. $p<0.001$ (two-way ANOVA). $n=5-28$ animals per time point. C, HFD $24+16$ mice gain more weight than their respective controls, and the percentage weight increase is significant starting from 3 weeks of HFD and onwards. $p<0.001$ (two-way ANOVA). $n=3-10$ animals per time point. $\boldsymbol{A}^{\prime}$, Terminal fasting blood glucose (FBG) at the experiment endpoint in HFD $4+16$ mice was not significantly different to the controls ( $n=5$ control, $n=8 \mathrm{HFD}$ ). $\boldsymbol{B}^{\prime}$, Terminal FBG in HFD $4+36$ mice was significantly higher than the controls. ${ }^{* *} p<0.01$ (unpaired two-tailed Student's $t$ test). $n=10$ control; $n=7$ HFD. $\boldsymbol{C}^{\prime}$, Terminal FBG in HFD $24+36$ mice
Western blot analysis

VDAC1/2, MFN2, and PGC1 $\alpha$ protein expression level was evaluated in the L1-L5 lumbar DRG or sural nerves from a separate cohort of mice fed either standard diet (Research Diets, D12450B) or 45\% HFD (Research Diets, D12451) from 4 to 36 weeks of age. Western blotting was conducted per our previous study (Vincent et al., 2010). Briefly, L1-L5 DRG or sural nerve from each mouse were pulverized with a pestle, resuspended in $50 \mu$ of RIPA lysis buffer (Sigma Millipore) with protease inhibitors (Roche), and evaluated for protein concentration. A total of $10 \mu \mathrm{g}$ of protein/lane was separated on a $12.5 \%$ polyacrylamide gel and transferred to a nitrocellulose membrane. Antibodies against PGC1 $\alpha$ (Abcam, catalog \#ab188102), MFN2 (Protein Tech, catalog \#12186-1-AP, RRID:AB 2266320), and VDAC1/2 (Protein Tech, catalog \#10866-1-AP, RRID:AB_2257153) were used to detect proteins. Membranes were then washed and probed for loading controls tubulin (Abcam, catalog \#ab6160, RRID:AB_305328) and YWHAZ (ProteinTech, catalog \#1 4881-1-AP, RRID:AB_2218248).

In vivo confocal image analyses

Mitochondrial movement was analyzed as described previously (Sajic et al., 2013) using the Difference Tracker plugin for ImageJ Fiji (SciJava) (Andrews et al., 2010). Mitochondrial size was evaluated in single images from a minimum of 5 axons from 5 or more nonoverlapping regions totaling at least 25 axon regions analyzed per mouse. Each single image showed 50-100 mitochondria, which

$\leftarrow$

was significantly higher than the controls. ${ }^{*} p<0.05$ (unpaired two-tailed Student's $t$ test). $n=8$ control; $n=7$ HFD. The impact of HFD on food consumption, FBG, body weight, and tactile response in male and female mice is shown in Extended Data Figure 1-2. $\boldsymbol{A}^{\prime \prime}$, ipGTT in HFD $4+16$ mice was significantly different to controls at all time points. ${ }^{*} p<0.05 ;{ }^{* *} p<0.01 ;{ }^{* * *} p<0.001$; Bonferroni post hoc test. $n=4$ or 5 per group. The overall effect from the HFD diet was extremely significant. $p<0.0001$ (two-way ANOVA). $\boldsymbol{B}^{\prime \prime}$, ipGTT in HFD $4+36$ mice was significantly different from controls at 60 and 120 min. ${ }^{* * *} p<0.001$ (Bonferroni post hoc test). $n=4-7$ per group. The overall effect from the HFD diet was extremely significant. $p<0.0001$ (two-way ANOVA). $C^{\prime \prime}$, ipGTT in HFD $24+16$ mice was significantly different from controls at 30,60, and 120 min. ${ }^{* * *} p<0.001$ (Bonferroni post hoc test). $n=10$ per group. The overall effect from the HFD diet was extremely significant. $p<0.0001$ (twoway ANOVA). D, Plasma cholesterol levels were significantly increased in HFD versus control $4+36$ mice. ${ }^{* * * *} p<0.0001$ (one-way ANOVA). $n=3$ per group. $\boldsymbol{E}$, Plasma phospholipid levels were significantly increased in HFD versus control $4+36$ mice. ${ }^{* * *} p<0.0001$ (oneway ANOVA). $n=3$ per group. $\boldsymbol{F}$, Nonesterified fatty acid (NEFA) levels did not differ in HFD versus control $4+36$ mice (one-way ANOVA, $n=3$ per group). $\mathbf{G}$, Triglyceride levels did not differ in HFD versus control $4+36$ mice (one-way ANOVA, $n=3$ per group). Quantitation of hairy skin and glabrous skin IENFD is shown in Extended Data Figure 1-3. 

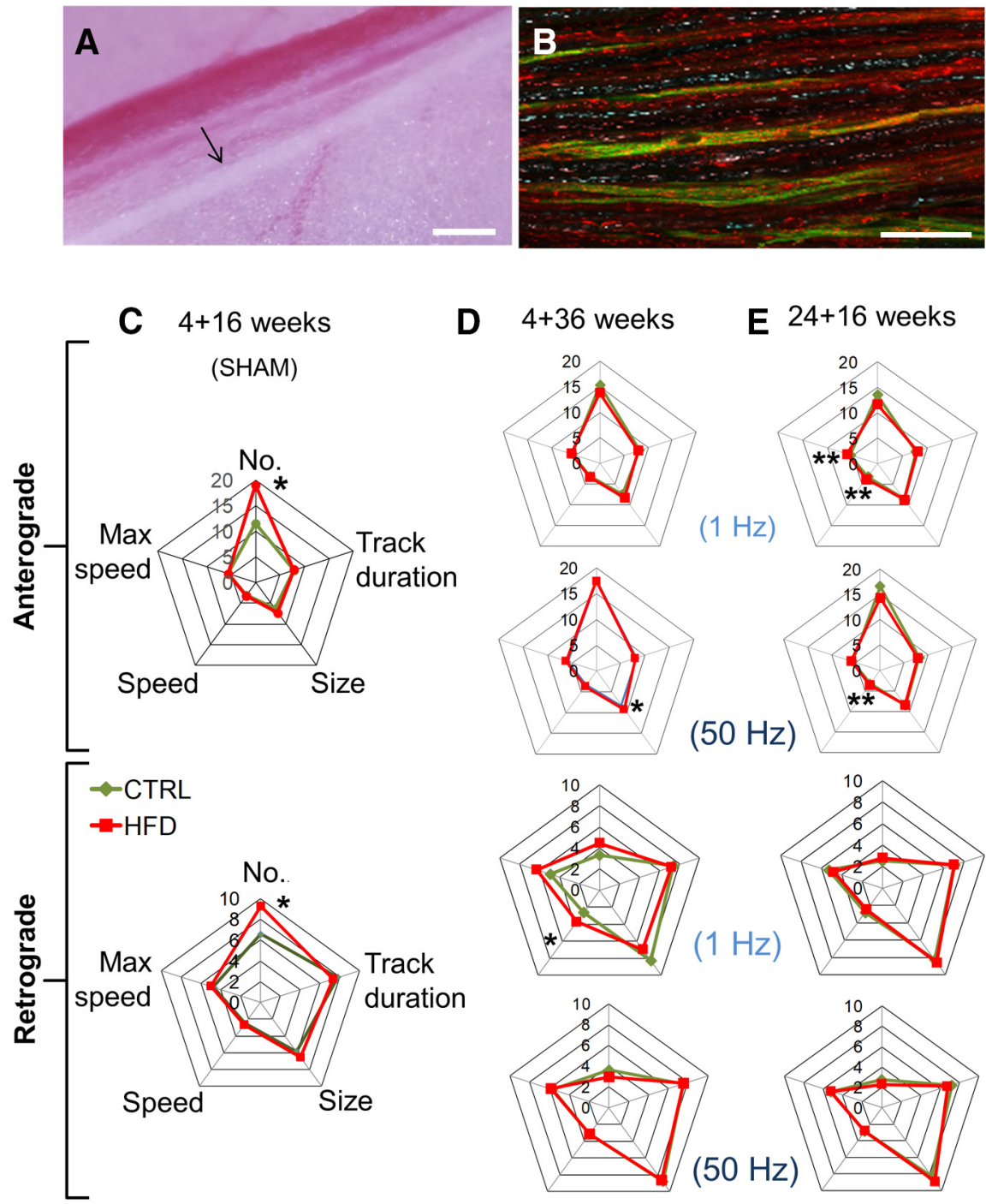

D $4+36$ weeks

E $24+16$ weeks
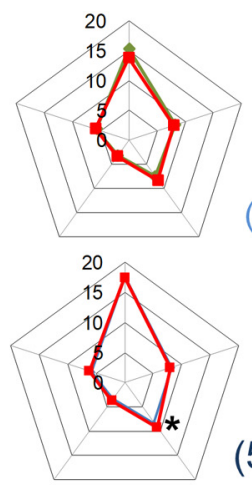

$(1 \mathrm{~Hz})$

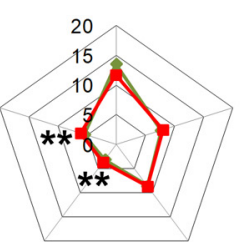

$(50 \mathrm{~Hz})$

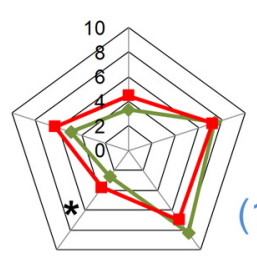

$(1 \mathrm{~Hz})$

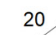

20

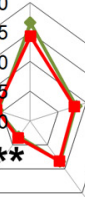

2
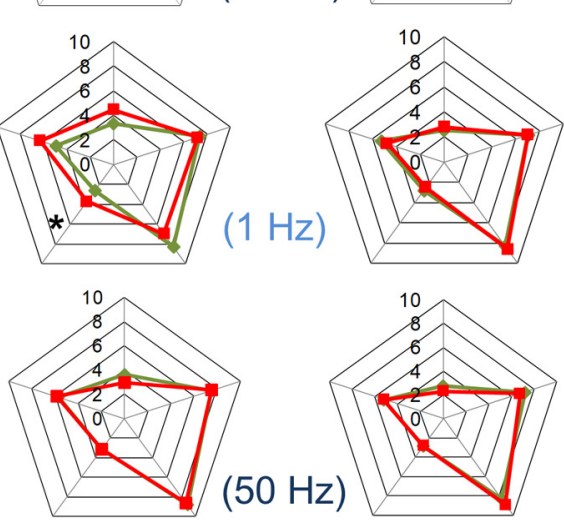

$(50 \mathrm{~Hz})$

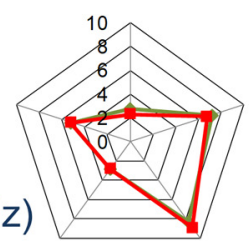

Figure 2. HFD mice do not exhibit impaired mitochondrial transport in saphenous nerve axons in vivo. $\boldsymbol{A}$, The saphenous nerve (black arrow), blood vessels, and the surrounding connective and fat tissue are exposed in the left thigh after skin incision. Scale bar, $1 \mathrm{~cm}$. B. Time-lapse high-magnification confocal image of saphenous nerve axons in CFP transgenic mouse with CFP ${ }^{+}$axonal mitochondria (blue). Isolectin-IB4 (green) labels a proportion of unmyelinated C-fibers and TMRM labels polarized mitochondria. Magenta, pink, or white represents $\mathrm{CFP}^{+}$, $\mathrm{TMRM}^{+}$mitochondria. Red represents $\mathrm{CFP}^{-}, \mathrm{TMRM}^{+}$mitochondria. Scale bar, $50 \mu \mathrm{m}$. C, Spider-web diagram showing characteristics of polarized, motile mitochondria in sham-stimulated saphenous nerve axons of HFD $4+16$ mice. There were significantly more motile anterograde and retrograde mitochondria in HFD than in control axons. $* p<0.05$ (two-way ANOVA). Data are mean of 37-66 axons per group for each category analyzed; $n=2$ mice per group. Videos of mitochondrial trafficking in control diet and HFD mice are shown in Movies 1 and 2, respectively. $\boldsymbol{D}, \boldsymbol{E}$, Spider-web diagrams showing characteristics of polarized, motile mitochondria in stimulated saphenous nerve axons of $4+36$ (D) or $24+16$ (E) mice. There were no overall differences in axonal mitochondrial transport. However, HFD $4+36$ mice exhibited larger anterogradely transported mitochondria in nerves conducting at $50 \mathrm{~Hz}$ and higher average retrograde speed at $1 \mathrm{~Hz} . * p<0.05$ (two-way ANOVA). Data are mean of 77-97 total axons per group; $n=3$ mice per group. HFD $24+16$ mice exhibited larger average speed of anterograde mitochondrial transport at both 1 and $50 \mathrm{~Hz}$ and higher anterograde maximum speed of mitochondrial transport at only $1 \mathrm{~Hz}$. $* * p<0.01$ (two-way ANOVA). Data are mean of 77-97 total axons per group; $n=3$ mice per group.

were analyzed by the Analyze Particle and Measure plugins for ImageJ, using the blue (CFP) channel, as described previously (Sajic et al., 2013). For the analysis of mitochondrial membrane potential, TMRM intensity was measured separately for every mitochondrion relative to its local background. First, the foreground TMRM pixels were identified from overlap with CFP pixels to identify axonal mitochondria. The median intensity of all foreground TMRM pixels of the particle was measured in ImageJ. Next, a local background selection was created containing all pixels within a 3 pixel radius around the mitochondrion of interest which were not part of, or immediately adjacent to, another mitochondrion, and the median intensity of these particles was calculated. The final intensity of a particle was defined as the median foreground intensity divided by the median background intensity. For analyzing $\left[\mathrm{Ca}^{2+}\right]_{\mathrm{i}}$, image analysis was performed using MATLAB (The MathWorks). First, all images were filtered using a $3 \times 3$ pixel median filter to reduce noise. Individual axons were extracted by manually selecting a rough axon outline. Then, all pixels of interest were selected by thresholding the anatomic (citrine-only) image. For each pixel of interest, the ratio of citrine to CFP in the ratiometric image was calculated. For the images of immunolabeled skin and DRG sections, $Z$ stacks were collapsed using Zen Black imaging software, which were opened in ImageJ. For the $\beta$-III tubulin-labeled images, dermis and epidermis in each image were carefully manually selected and the area measured using the Measure plugin for ImageJ. The images were then carefully thresholded to ensure that the resulting image faithfully represented the original image. The number of labeled pixels was determined using the Measure plugin for Image $J$ and expressed per normalized area unit. For the analysis of PGC1 $\alpha$ labeling in DRGs, and $\beta$-III tubulin in the skin, three cells in each FOV, with a minimum 3 FOVs per animal, were randomly chosen from the collapsed $Z$ stacks. The cell area was carefully outlined and measured using ImageJ. Images were carefully thresholded to ensure that the resulting image faithfully reproduced the original image, and areas of PGC1 $\alpha$ and $\beta$-III tubulin labeling were measured again, and expressed as percentage of the cell or skin area, respectively.

\section{Data analysis}

Before all statistical analyses, the data were tested for normality of distribution using D'Agostino and Pearson normality test with Prism version 5 (GraphPad Software). Differences between groups in weight, food consumption, ipGTT, hyperglycemia, tactile hypersensitivity, and all parameters of mitochondrial dynamics and size, and amplitudes of second response in RPT were calculated using two-way ANOVA with Bonferroni's multiple comparison tests. Two-tailed Student's $t$ test was used for analyzing differences in terminal blood glucose, MMP, and loss of amplitude after $1 \mathrm{~h}$ conduction at $50 \mathrm{~Hz}$ in $\mathrm{HFD}$ versus control mice. Mann-Whitney test was applied to analyze differences in $\left[\mathrm{Ca}^{2+}\right]_{\mathrm{i}}$ in HFD $9+5$ mice and $\mathrm{PGC} 1 \alpha$ and $\beta$-III tubulin coverage. Finally, Kruskal-Wallis test was used to analyze differences between groups in $\left[\mathrm{Ca}^{2+}\right]_{\mathrm{i}}$ in mice fed HFD for 16 weeks starting from 24 weeks of age. An ordinary one-way ANOVA was used to evaluate complex lipid panel data. A two-tailed unpaired $t$ test was used to assess VDAC1/ 2, MFN2, and PGC1 $\alpha$ protein expression level in DRG and sural nerves. Parametric data are presented as mean \pm SEM, whereas nonparametric data, or data that did not follow a normal distribution, are presented as median \pm interquartile range. A $p$ value $<0.05$ was considered statistically significant. 


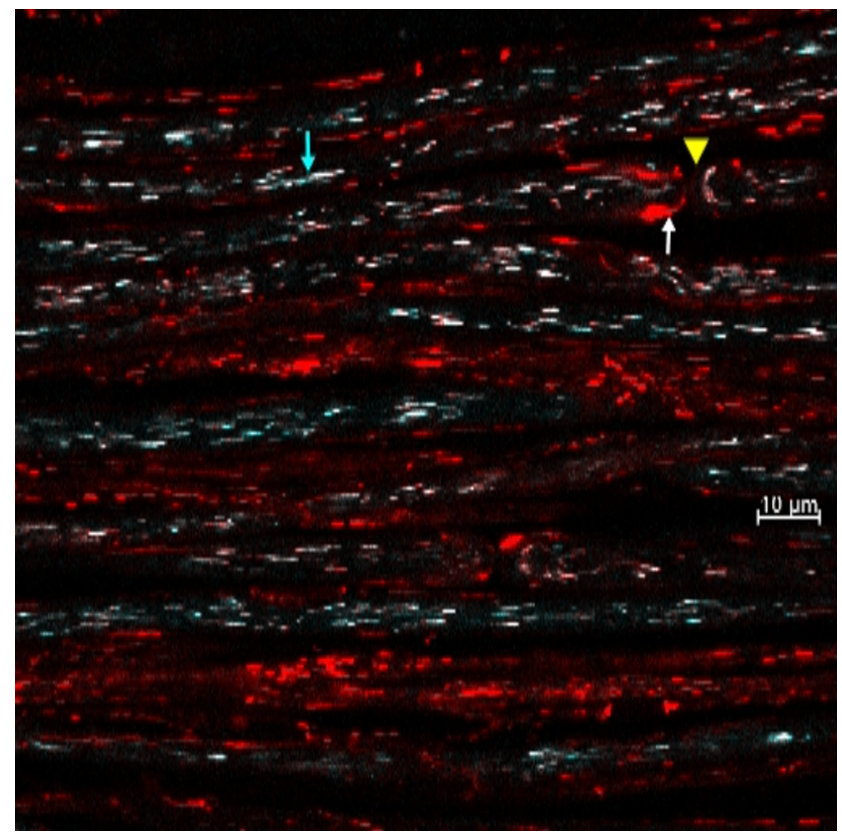

Movie 1. Time-lapse video of axonal mitochondrial trafficking in a control mouse in vivo in saphenous nerve conducting at $1 \mathrm{~Hz}$. High-magnification, time-lapse confocal image of saphenous nerve axons in a control $24+16$ CFP transgenic mouse with $\mathrm{CFP}^{+}$axonal mitochondria (blue) stimulated at $1 \mathrm{~Hz}$. TMRM labels polarized mitochondria (magenta), (FP ${ }^{+}$ (pink), $\mathrm{TMRM}^{+}$(white) (blue arrow). CFP', TMRM ${ }^{+}$Schwann cell mitochondria are red (white arrow). Relative to the imaging site, axons were stimulated at $1 \mathrm{~Hz}$ proximally (left of the image) and conducted compound action potentials were recorded distally (right of the image). Many motile mitochondria move in either direction. A node of Ranvier is present in the upper part of the image (yellow arrowhead). Scale bar, $10 \mu \mathrm{m}$. [View online]

\section{Results}

HFD mice exhibit weight gain and impaired glucose tolerance

We examined the effect of HFD on mitochondrial trafficking and function in vivo in three experimental groups of mice fed a HFD (45\% calories from fat), compared with their respective controls. The first and second groups consisted of "young" mice fed a short- (16 weeks) or long-term (36 weeks) diet, starting at 4 weeks of age (i.e., HFD $4+16$ and HFD $4+36$ groups, respectively) (Fig. 1, top). The third group consisted of "older" mice fed short-term (16 weeks) HFD starting from 24 weeks of age (HFD $24+16$ group). Corresponding control littermates received control diet (13\% calories from fat) for the same duration as their HFD counterparts. HFD mice gained significantly more weight than their control counterparts (Fig. $1 A-C$ ). Furthermore, mice from the HFD $4+36$ and $24+16$ groups developed pronounced hyperglycemia compared with control mice (Fig. $1 B^{\prime}, C^{\prime}$ ), whereas glucose tolerance was impaired across all HFD groups (Fig. $1 A^{\prime \prime}-C^{\prime \prime}$ ). Both HFD and control mice consumed the same amount of food per day, suggesting that the changes to body weight and hyperglycemia are related to diet composition rather than differences in food intake (Extended Data Fig. 1-2). We next assessed the effect of HFD on circulating lipids. Compared with control mice, HFD $4+36$ mice had elevated plasma cholesterol and phospholipids levels, but no differences in triglycerides and nonesterified fatty acid levels (Fig. $1 D-G$ ). Finally, we evaluated the PN phenotype by quantifying IENFD, which was reduced in glabrous skin of HFD versus control mice (Extended Data Fig. 1-3). Our combined findings show that HFD mice recapitulate metabolic dysfunction in prediabetic patients.

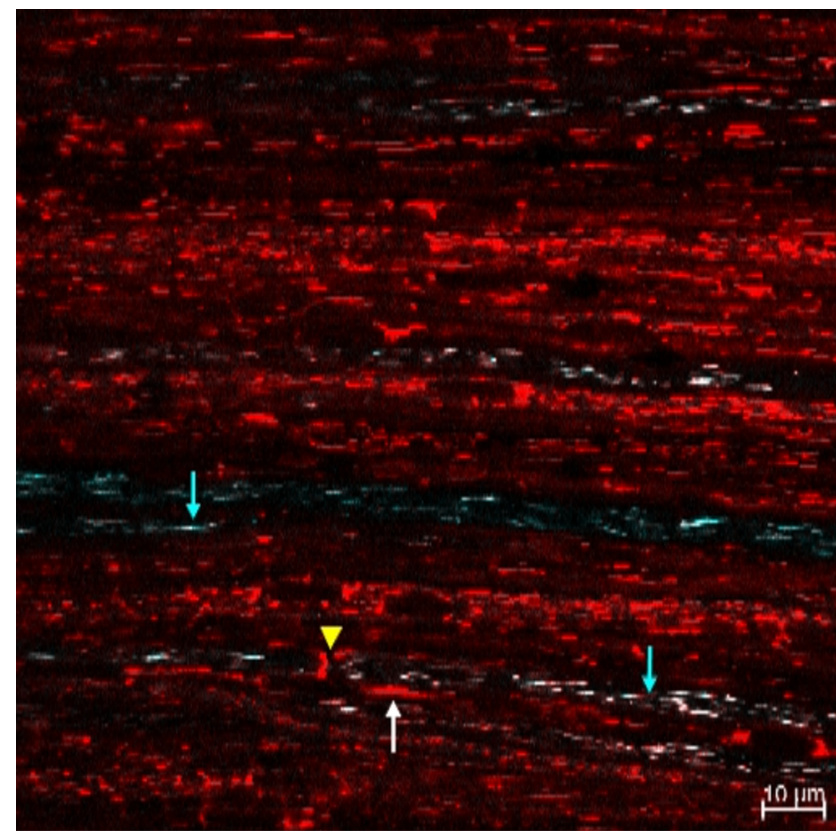

Movie 2. Time-lapse video of axonal mitochondrial trafficking in an HFD mouse in vivo in saphenous nerve conducting at $50 \mathrm{~Hz}$. High-magnification, time-lapse confocal image of saphenous nerve axons in a control $24+16$ CFP transgenic mouse with CFP $^{+}$axonal mitochondria (blue) stimulated at $50 \mathrm{~Hz}$. TMRM labels polarized mitochondria (red or magenta), $\mathrm{CFP}^{+}, \mathrm{TMRM}^{+}$and depolarised, $\mathrm{CFP}^{+} \mathrm{TMRM}^{-}$(blue) (blue arrow). CFP ${ }^{-}, \mathrm{TMRM}^{+}$Schwann cell mitochondria are red (white arrow). Relative to the imaging site, axons were stimulated at $50 \mathrm{~Hz}$ proximally (left of the image) and conducted compound action potentials were recorded distally (right of the image). Many motile mitochondria move in either direction. A node of Ranvier is present in the lower part of the image (yellow arrowhead). Scale bar, 10 $\mu \mathrm{m}$. [View online]

An additional cohort of HFD female mice developed hyperglycemia and gained a similar amount of weight to HFD male mice (Extended Data Fig. 1-2), although HFD females had a higher percent increase in body weight versus HFD males starting at 22 weeks. Male and female mice displayed similar tactile hypersensitivity at 20 and 40 weeks, when in vivo mitochondrial dynamics were measured, although development was slightly delayed in females (Extended Data Fig. 1-2). Therefore, in vivo mitochondrial analyses were only performed in male mice.

\section{HFD mice do not exhibit impaired mitochondrial transport in saphenous nerve axons in vivo}

Terminal mitochondrial transport was examined in adult, electrically active, myelinated saphenous nerve axons in vivo (Fig. 1, top). In this transgenic mouse, $\sim 40 \%-60 \%$ of axonal mitochondria constitutively express CFP $\left(\mathrm{CFP}^{+}\right.$, Fig. $\left.2 A, B\right)$ (Sajic et al., 2013). Nerves were also stained in situ with TMRM, a cationic dye that accumulates in mitochondria in proportion to their MMP, staining them red. $\mathrm{CFP}^{+}$, unpolarized mitochondria (do not stain with TMRM) will appear cyan, whereas $\mathrm{CFP}^{+}$, polarized mitochondria (stain with TMRM) will appear magenta, pink, or white. Unpolarized mitochondria that do not express $\mathrm{CFP}\left(\mathrm{CFP}^{-}\right)$will not be visible, whereas polarized, $\mathrm{CFP}^{-}$mitochondria will be red. At the experimental endpoint, we used time-lapse confocal microscopy to track CFP (axonal mitochondria; nonaxonal mitochondria do not express CFP) and TMRM (polarized) labeled motile mitochondria (Movies 1 and 2).

Sham-stimulated axons, which are presumed to be electrically silent, had a significantly greater number of motile mitochondria in both anterograde and retrograde directions in HFD $4+16$ 

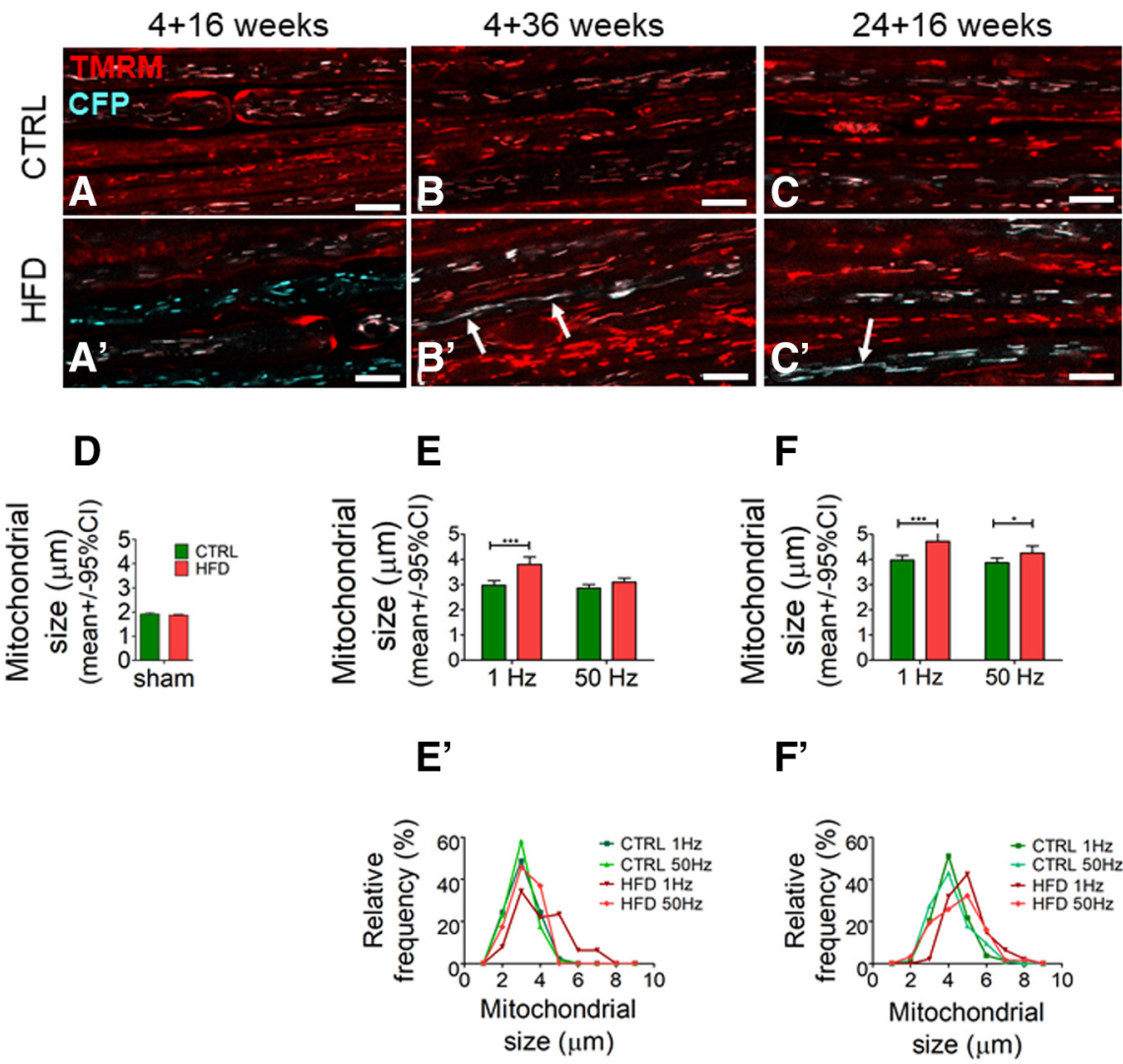

Figure 3. HFD mice exhibit increased stationary mitochondrial size in saphenous nerve axons in vivo. $\boldsymbol{A}-\boldsymbol{C}, \boldsymbol{A}^{\prime}-\boldsymbol{C}^{\prime}$, Highmagnification confocal image of saphenous nerve axons in (FP transgenic mouse with $\mathrm{CFP}^{+}$axonal mitochondria (blue) stimulated at $1 \mathrm{~Hz}$. TMRM labels polarized mitochondria. Magenta, pink, or white represents CFP $^{+}, \mathrm{TMRM}^{+}$mitochondria. Red represents CFP $^{-}$, $\mathrm{TMRM}^{+}$mitochondria. Scale bar, $10 \mu \mathrm{m}$. $\boldsymbol{A}-\boldsymbol{C}$, In saphenous nerve from control $4+16(\boldsymbol{A}), 4+36$ $(\boldsymbol{B})$, and $24+16(\boldsymbol{C})$ mice. $\boldsymbol{A}^{\prime}-\boldsymbol{C}^{\prime}$, In saphenous nerve axons from HFD $4+16\left(\boldsymbol{A}^{\prime}\right), 4+36\left(\boldsymbol{B}^{\prime}\right)$, and $24+16\left(\boldsymbol{C}^{\prime}\right)$ mice. Several axonal mitochondria were longer in older HFD (white arrows) than in control mice. $\boldsymbol{D}$, HFD $4+16$ mice had similar average stationary mitochondrial size at the experiment endpoint in their saphenous nerve axons compared with controls (mean \pm SEM, $n=2$ mice per group, $n=2996$ control mitochondria, $n=9987$ HFD mitochondria). $\boldsymbol{E}, \boldsymbol{F}$, HFD $4+36$ and $24+16$ mice had increased average stationary mitochondrial size at the experiment endpoint in their saphenous nerve axons stimulated at $1 \mathrm{~Hz}$ and for HFD $24+16$ mice also at $50 \mathrm{~Hz}$ versus controls. ${ }^{*} p<0.05 ;{ }^{* * *} p<0.001$; two-way ANOVA. Data are mean \pm SEM. $n=4$ or 5 mice per group. $\boldsymbol{E}^{\prime}, \boldsymbol{F}^{\prime}$, Distribution of stationary mitochondrial size at the experiment endpoint in saphenous nerve axons shows a shift toward larger mitochondria in $4+36$ and $24+16$ HFD versus control mice.

mice than corresponding controls (Fig. 2C), while other parameters of mitochondrial motility and size were similar. We then analyzed mitochondrial transport in mice fed long-term HFD $(4+36$ mice, Fig. $2 D)$, or in older mice fed the short-term HFD $(24+16$ mice, Fig. 2E; Movies 1 and 2). In addition, to mimic physiological axonal activity in live animals, we electrically stimulated saphenous nerve axons at either low $(1 \mathrm{~Hz})$ or high $(50 \mathrm{~Hz})$ frequency during imaging. Electrical stimulation places an energy demand on axons; thus, mitochondria mobilize in response. However, regardless of the impulse load on the axons, we found no difference in the number of motile mitochondria between the groups (Fig. 2D,E). Detailed analysis showed that HFD $4+36$ mice (Fig. 2D) exhibited a significant increase in the size of anterogradely transported mitochondria in nerves conducting at $50 \mathrm{~Hz}$, and in the average speed of retrogradely transported mitochondria in nerves conducting at $1 \mathrm{~Hz}$, in comparison with controls. Also, in HFD $24+16$ mice (Fig. $2 E$ ), the average speed of anterograde mitochondrial transport was significantly higher than in control nerves, at both 1 and $50 \mathrm{~Hz}$, and the anterograde maximal speed of mitochondrial transport was significantly higher versus controls at only $1 \mathrm{~Hz}$. Overall, however, our results indicate that, in contrast to our expectations from in vitro data (Rumora et al., 2018, 2019a), prediabetic mice do not exhibit impaired mitochondrial transport along saphenous nerve axons in vivo. Although there was a small increase in the number, size, and speed of mitochondria in some HFD groups, it was not sufficiently pronounced to affect overall axonal mitochondrial transport.

\section{HFD mice exhibit increased stationary mitochondrial size in saphenous nerve} axons in vivo

In healthy axons, the majority of axonal mitochondria remain stationary regardless of the level of impulse activity. However, stationary mitochondria in electrically stimulated axons are shorter than mitochondria in electrically silent or low-frequency conducting axons. This finding has been interpreted as activity-induced mitochondrial fission intended to generate smaller more easily transportable mitochondria (Sajic et al., 2013). To examine the effect of HFD on stationary, polarized mitochondria in vivo, we next measured their size by confocal microscopy (Fig. 3). There were no differences in the length of stationary mitochondria in young mice fed a short 16 week HFD $(4+16)$ versus control diet (Fig. 3A,D). However, in mice fed HFD for 36 weeks, stationary mitochondria in axons conducting at $1 \mathrm{~Hz}$ were significantly longer compared with control mice (Fig. 3B,E). Further, the average size of stationary mitochondria was significantly longer in older mice fed HFD $(24+16)$ versus controls at both frequencies (Fig. 3C,F). To illustrate the change in the size distribution of stationary mitochondria, we stratified the average size data (Fig. $3 E^{\prime}, F^{\prime}$ ) and presented them as a proportion of all stationary axonal mitochondria (Fig. $3 B^{\prime}$, $\left.C^{\prime}\right)$. A trend toward a greater proportion of larger (i.e., longer than $5 \mu \mathrm{m}$ ) versus shorter stationary mitochondria was found in mice fed HFD versus control diet in the $4+36$ and $24+16$ week groups.

\section{HFD-fed mice exhibit increased PGC1 $\alpha$ expression in DRGs in vivo}

The transcriptional coactivator $\mathrm{PGC} 1 \alpha$ is a master regulator of mitochondrial biogenesis, fatty acid oxidation (Scarpulla, 2011), and intracellular calcium levels (Chen et al., 2010). Thus, we next sought to determine whether PGC1 $\alpha$ levels are altered in peripheral nerves of HFD mice. We used confocal fluorescence microscopy to quantify $\mathrm{PGC} 1 \alpha$ protein expression in spinal cord tissue sections from the lumbar L3 and L4 segments by immunohistochemistry. The HFD $4+36$ mice displayed elevated PGC1 $\alpha$ immunoreactivity compared with controls in both L3 (Fig. 4A-C) and L4 DRGs (Fig. 4D-F). PGC1 $\alpha$ protein level in lumbar DRG was also significantly increased in HFD versus control mice in a separate cohort fed respective diets from 4 to 36 weeks (Fig. $4 G$ ). 
PGC1 $\alpha$ protein level did not differ in HFD versus control sural nerves (Fig. $4 H$ ). Although PGC1 $\alpha$ can trigger MFN2-mediated mitochondrial fusion, there was no difference in DRG MFN2 protein levels in HFD and control mice (Extended Data Fig. 4-1). VDAC1/2, an important regulator of mitochondrial function, was significantly decreased in HFD sural nerves, but unchanged in HFD DRG (Extended Data Fig. 4-1). Therefore, increased PGC1 $\alpha$ expression occurred concomitantly with increased mitochondrial length (Fig. 3), and reduced mitochondrial MMP (Fig. 5) and $\left[\mathrm{Ca}^{2+}\right]_{i}$ in HFD saphenous nerve axons (Fig. 7).

HFD mice exhibit reduced MMP in saphenous nerve axons in vivo MMP is a key requirement for mitochondrial ATP synthesis (Zorova et al., 2018). We showed previously that SFAs decrease MMP in primary DRG neurons in vitro (Rumora et al., 2018, 2019a). Therefore, we next examined whether HFD impaired MMP in vivo in axons under conditions of both low and high energy demand. We quantified MMP in vivo by acquiring confocal images of TMRM-labeled $\mathrm{CFP}^{+}$ axons and calculating the ratio of TMRM intensity in $\mathrm{CFP}^{+}$mitochondria versus TMRM intensity in the adjacent surrounding axoplasm. To monitor MMP within electrically active axons, we imaged saphenous nerve axons while simultaneously stimulating them. In sham-stimulated (i.e., basal energy demand) HFD $4+16$ and $1 \mathrm{~Hz}$-stimulated (i.e., low energy demand) HFD $4+36$ and $24+16$ saphenous nerve axons, mitochondria stained more weakly with TMRM than in control mice, indicating lower MMP in HFD mitochondria (Fig. $5 A, A^{\prime}, B, C$ ). We next increased axonal energy demand by stimulating the saphenous nerves at $50 \mathrm{~Hz}$, which markedly decreased MMP in control mitochondria (Fig. $5 B^{\prime}, C^{\prime}$ ). This is consistent with the basic tenet of mitochondrial function whereby MMP (the proton gradient) is dissipated through complex $\mathrm{V}$ to generate ATP. In contrast, in mice fed HFD, conducting at $50 \mathrm{~Hz}$ did not change MMP. Overall, this suggests that the lower MMP in HFD mice may suffice to meet basal energy demands for axon survival, but not for meeting the energy demands for the full range of physiological functions, including high-frequency impulse activity.

\section{HFD mice exhibit impaired axonal conduction in saphenous} nerve axons in vivo

Impulse conduction requires increased ATP production (van Hameren et al., 2019); however, we found herein that HFD mitochondria fail to dissipate MMP in response to electrical stimulation (Fig. 5), suggesting that axons from HFD mice may not be able to conduct at the physiological frequencies required for normal functioning (e.g., sensory perception during walking) (Prochazka and Gorassini, 1998). Therefore, we examined whether HFD saphenous nerve axons could sustain conduction at physiological frequency in vivo by measuring sCAPs (i.e., simultaneous action potentials from several axons). Low-frequency $(1 \mathrm{~Hz})$ stimulation generated synchronous sCAPs from HFD mice saphenous axons that were comparable to those in control mice (Extended Data Fig. 6-1). Only in some mice on long-term HFD ( $4+36$ weeks) were sCAPs at $1 \mathrm{~Hz}$ more dispersed, indicating slowing of conduction velocity in some axons (Extended Data Fig. 6-1). These findings suggest that, although MMP was significantly lower in HFD versus control axons, HFD mitochondria still generate sufficient ATP to conduct impulses at $1 \mathrm{~Hz}$. Importantly, however, such low level of impulse activity 


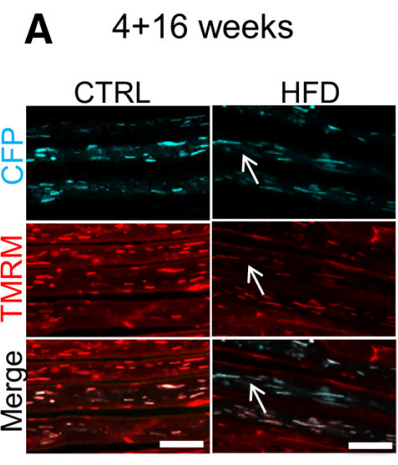

B $4+36$ weeks

\section{C $24+16$ weeks}
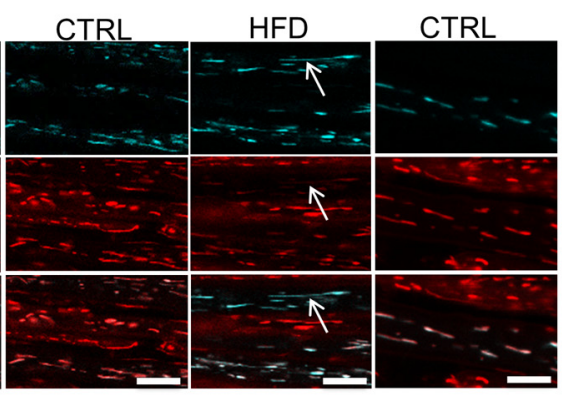

$A^{\prime}$

B'
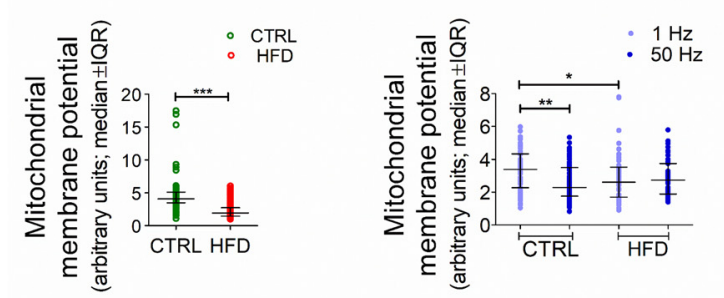

$C^{\prime}$

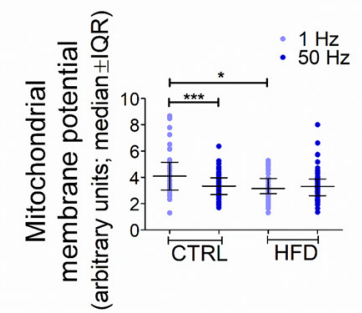

Figure 5. HFD mice exhibit reduced MMP in saphenous nerve axons in vivo. $\boldsymbol{A}-\boldsymbol{C}$, High-magnification confocal image of saphenous nerve axons in CFP transgenic mouse with $\mathrm{CFP}^{+}$axonal mitochondria (blue, top) stimulated at $1 \mathrm{~Hz}$. TMRM labels polarized mitochondria (red, middle). Bottom, Merged. Magenta, pink, or white represents CFP $^{+}, \mathrm{TMRM}^{+}$mitochondria. Red represents $\mathrm{CFP}^{-}, \mathrm{TMRM}^{+}$mitochondria. In control mice, all axonal mitochondria were polarized and were magenta, pink, or white on merged images. Bottom, Red mitochondria are axonal, non-CFP expressing, or nonaxonal. In contrast, in all HFD groups, some axonal mitochondria were depolarized and appear blue on merged images (bottom, white arrows). $\boldsymbol{A}^{\prime}$, The ratio of TMRM intensity in CFP ${ }^{+}$mitochondria versus the adjacent axoplasm indicates MMP and is significantly lower in HFD $4+16$ mice versus controls. ${ }^{* *} p<0.001$ (unpaired Student's $t$ test). Data are mean \pm SEM. $n=4$ or 5 mice per group. $\boldsymbol{B}^{\prime}$, The ratio of TMRM intensity in $\mathrm{CFP}^{+}$mitochondria versus the adjacent axoplasm indicates MMP and is significantly lower in HFD $4+36$ mice versus controls in $1 \mathrm{~Hz}$-stimulated axons. Increasing the stimulation frequency to $50 \mathrm{~Hz}$ lowers MMP in control mitochondria but has no effect in HFD mitochondria. ${ }^{*} p<0.05$; ${ }^{* *} p<0.01$; ${ }^{* * *} p<0.001$; two-way ANOVA. Data are mean \pm SEM. $n=4$ or 5 mice per group. $\boldsymbol{C}^{\prime}$, The ratio of TMRM intensity in CFP $^{+}$mitochondria versus the adjacent axoplasm indicates MMP and is significantly lower in HFD $24+16$ mice versus controls in axons stimulated at $1 \mathrm{~Hz}$. Increasing the stimulation frequency to $50 \mathrm{~Hz}$ lowers MMP in control mitochondria but has no effect in HFD mitochondria. ${ }^{*} p<0.05$; ${ }^{* *} p<0.01$; ${ }^{* * *} p<0.001$; two-way ANOVA. Data are mean \pm SEM. $n=4$ or 5 mice per group. IQR, Interquartile range.

is atypical for nonresting sensory axons, which require sustained impulse conduction between 50 and $150 \mathrm{~Hz}$ for moderate physical activity, such as walking.

Thus, we repeated sCAP recordings during $50 \mathrm{~Hz}$ electrical stimulation. In control mice, sCAPs maintained the same shape and amplitude for at least $60 \mathrm{~min}$ (Fig. 6A,D); however, sCAP amplitudes steadily declined in both $4+36$ (Fig. 6B,D) and $24+$ 16 (Fig. 6C,D) HFD mice. The reduced sCAP amplitudes were sometimes accompanied by the appearance of additional, late peaks (Fig. 6B) and an overall latency increase (Fig. 6C). The reduced amplitudes during sustained impulse activity were more pronounced in animals fed HFD for a longer period. After 16 weeks of HFD diet, the average sCAP amplitude dropped to $80 \pm 19 \%$ (mean $\pm S D$ ) of the initial amplitude (Fig. $6 C^{\prime}$ ), significantly less than in controls ( $p=0.03$, unpaired Student's $t$ test). After 36 weeks on HFD, the sCAP amplitudes dropped to as little as only $27 \%$ in some animals, with a trending average drop of $71 \pm 34 \%$ of the initial amplitude (Fig. $\left.6 B^{\prime}\right)(p=0.10$, unpaired Student's $t$ test).

To explore the extent of PN in these mice, we next measured their axonal RPT (Smith, 1980), a sensitive method for detecting early PN (Braune, 1999). RPT is the period after one action potential has propagated that the axon is refractory to the propagation of a second action potential. We quantified RPT in HFD

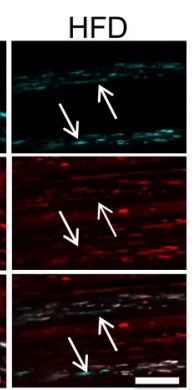

and control axons by delivering a first stimulus and increasing the delay a second stimulus was applied in steps of $0.1 \mathrm{~ms}$ up to a $2.5 \mathrm{~ms}$ delay (Fig. $6 E, F$ ). We quantified the percentage of axons that conducted the second stimulus at each stimulus interval (Fig. 6G,H). In control nerves, some axons were still able to conduct the second stimulus after a delay of only $0.3 \mathrm{~ms}$ (Fig. 6E, green arrow), $\sim 50 \%$ still conducted after a $0.8 \mathrm{~ms}$ delay, and $80 \%$ after a 1.2 ms delay (Fig. 6H). In contrast, in HFD $24+16$ mice, no axons conducted after a $0.3 \mathrm{~ms}$ delay, only $\sim 10 \%$ conducted after a 0.8 ms delay, and $\sim 50 \%$ conducted after a $1.2 \mathrm{~ms}$ (Fig. $6 H$ ). These results confirm that 16 weeks on HFD is sufficient to detectably impair conduction. Interestingly, after a longer 36 week HFD duration, RPT tended to revert toward the control pattern (Fig. 6G). Nonetheless, these mice showed greater deterioration in their ability to conduct at $50 \mathrm{~Hz}$ compared with mice on a shorter diet duration (Fig. $6 B^{\prime}, C^{\prime}$ ). Overall, our data show that the loss of MMP coincides with the loss of axonal conduction in HFD mice.

\section{HFD mice exhibit reduced $\left[\mathrm{Ca}^{2+}\right]_{\mathrm{i}}$} levels in saphenous nerve axons in vivo Since HFD causes axonal mitochondrial deficits, and mitochondria are important regulators of $\left[\mathrm{Ca}^{2+}\right]_{\mathrm{i}}$ (Rizzuto et al., 2012), we next examined whether HFD alters $\left[\mathrm{Ca}^{2+}\right]_{\mathrm{i}}$ in saphenous nerve axons in vivo. We used the transgenic Tn-XXL mouse, which expresses a calcium-binding troponin C-based biosensor with two fluorescent probes, citrine and CFP (Mank et al., 2008). Calcium binding to the sensor results in fluorescence resonance energy transfer (FRET); thus, $\left[\mathrm{Ca}^{2+}\right]_{i}$ can be quantified by taking the ratio of citrine to CFP fluorescence intensity from axons imaged by confocal microscopy (Fig. 7A-C). We first validated our system by incubating saphenous nerve axons in vivo in Tn-XXL mice in aCSF followed by adding ionomycin, an ionophore that raises $\left[\mathrm{Ca}^{2+}\right]_{\mathrm{i}}$. As anticipated, the citrine to CFP fluorescence intensity ratio increased (Fig. 7D).

In preliminary experiments, we examined the consequences of a relatively short duration of HFD (5 weeks) on $\left[\mathrm{Ca}^{2+}\right]_{\mathrm{i}}$, using 13 -week-old Tn-XXL mice ( $13+5$ group). This regimen only caused a moderate body weight increase $(22.23 \pm 1.13 \mathrm{~g}$ in control vs $26.13 \pm 0.83 \mathrm{~g}$ in HFD), yet, surprisingly, it significantly reduced $\left[\mathrm{Ca}^{2+}\right]_{\mathrm{i}}(p=0.0005$, Mann-Whitney test, $n=4$ animals per group; Fig. 7 C). We examined $\left[\mathrm{Ca}^{2+}\right]_{\mathrm{i}}$ in HFD $24+16$ mice, given that this cohort displayed significantly impaired axonal conduction (Fig. 6). This HFD regimen in Tn-XXL mice induced progressive metabolic dysfunction, including accelerated weight gain, hyperglycemia, and glucose intolerance (Extended Data Fig. 7-1), similar to the effects seen in CFP mice (Fig. 1). Interestingly, unmyelinated axons (C-fibers, distinguishable by caliber $<1.5 \mu \mathrm{m}$ ), which are typically affected early in the course of $\mathrm{PN}$ and manifest as pain, showed significantly greater $\left[\mathrm{Ca}^{2+}\right]_{\mathrm{i}}$ 


\section{$50 \mathrm{~Hz}$ conduction for 1 hour}
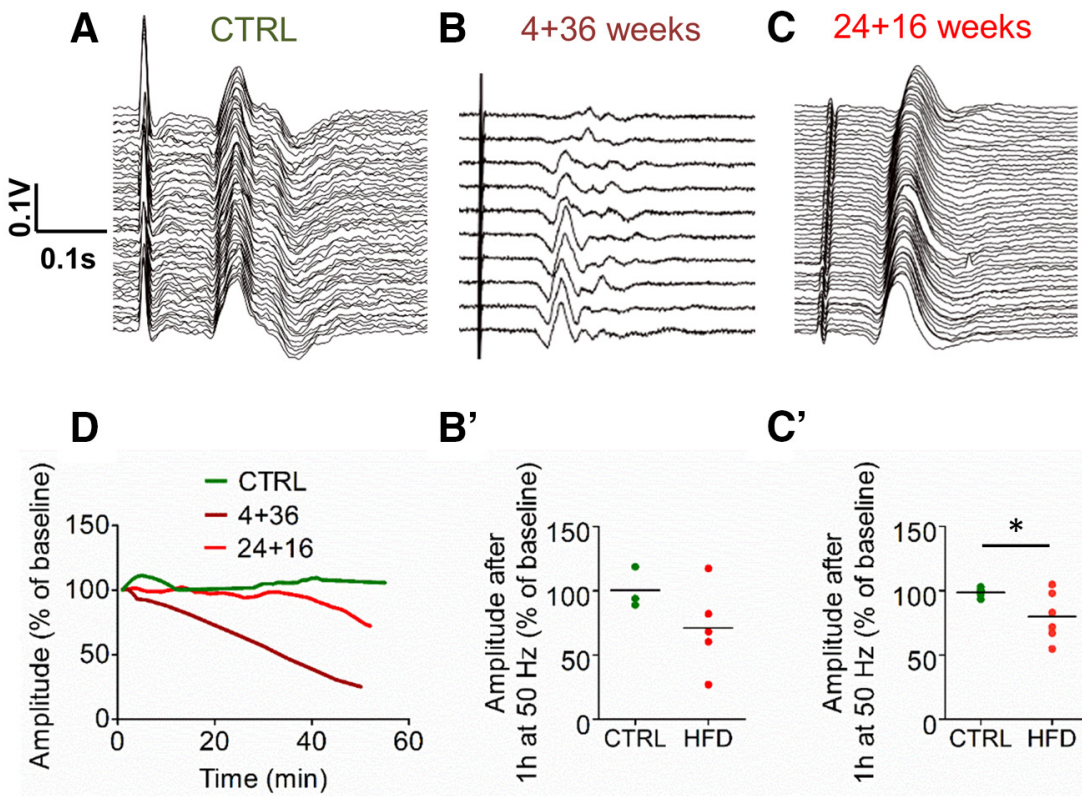

B'

$C^{\prime}$
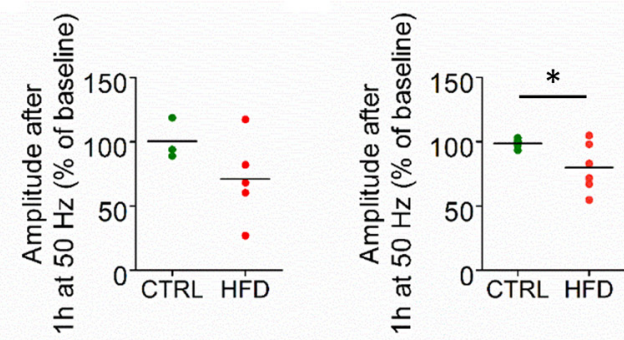

Refractory period
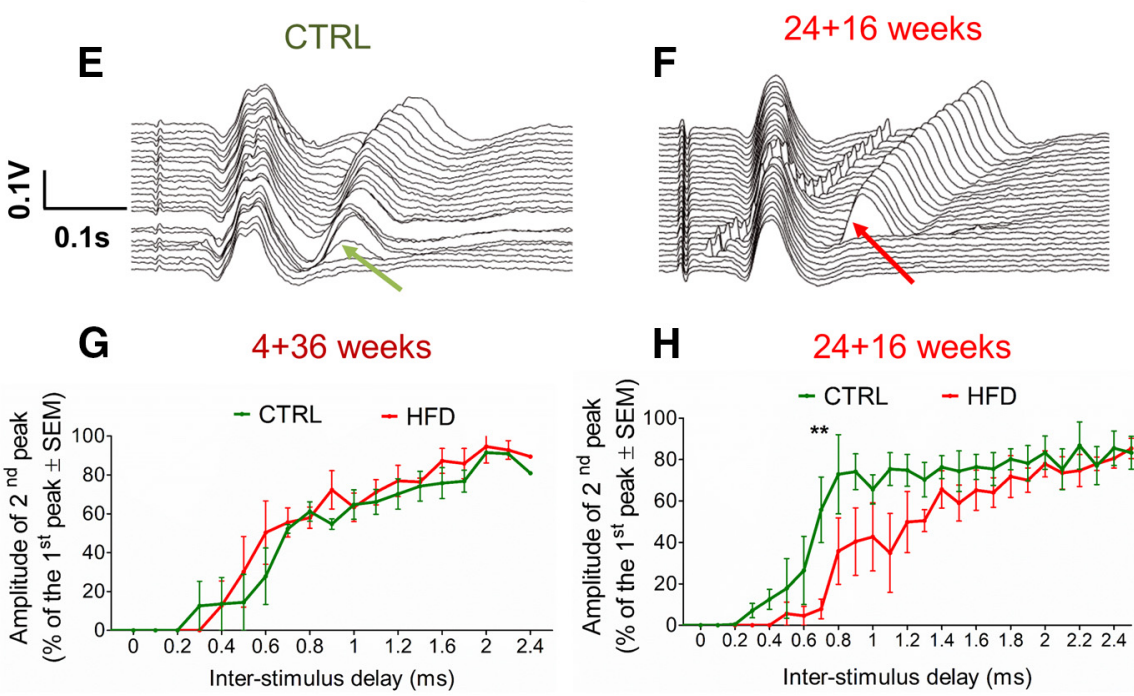

Figure 6. HFD mice exhibit impaired axonal conduction in saphenous nerve axons in vivo. A, Waterfall graph of sCAPs from a control mouse, which remain relatively unchanged over $1 \mathrm{~h}$ of sustained impulse activity at $50 \mathrm{~Hz}$. The earliest records are at the front of this plot and all other waterfall plots. $\boldsymbol{B}$, Waterfall graph of sCAPs from a $4+36$ HFD mouse, which progressively change over $1 \mathrm{~h}$ of sustained impulse activity at $50 \mathrm{~Hz}$. The records show a loss of amplitude over time and the labile appearance of axons conducting at longer latency. $\boldsymbol{B}^{\prime}$, Plot of change in sCAP amplitude after $1 \mathrm{~h}$ of conducting at $50 \mathrm{~Hz}$, expressed as a percentage of the starting amplitude. There is no mean loss of amplitude in controls, but a mean reduction of $29 \%$ in HFD $4+36$ mice. $p=0.05$ (unpaired Student's $t$ test). $n=3$ control; $n=5$ HFD. Example traces of conduction at $1 \mathrm{~Hz}$ in HFD and control $4+36$ mice are shown in Extended Data Figure 6-1. C, Waterfall graph of sCAPs from a $24+16 \mathrm{HFD}$ mouse, which progressively change over $1 \mathrm{~h}$ of sustained impulse activity at $50 \mathrm{~Hz}$. The records show a loss of amplitude, indicative of conduction block or partial depolarization, and increase in latency, indicating conduction slowing. $\mathbf{C}^{\prime}$, Plot of change in SCAP amplitude after $1 \mathrm{~h}$ of conducting at $50 \mathrm{~Hz}$, expressed as a percentage of the starting amplitude. There is no mean loss of amplitude in controls, but a mean reduction of 20\% in HFD $24+16$ mice. $p=0.03$ (unpaired Student's $t$ test). $n=3$ control; $n=6 \mathrm{HFD}$. D, Loss of sCAP amplitude over time in a control (green), $4+36$ HFD (dark red), and $24+16$ HFD (red) mouse, showing dramatic loss in \% of axons conducting $50 \mathrm{~Hz}$ in HFD mice. $\boldsymbol{E}$, Waterfall graph of RPTs in a control mouse, revealing that a proportion of axons can still conduct a second stimulus delivered $0.3 \mathrm{~ms}$ after the first (green arrow); the proportion of axons that conduct the second stimulus increases as the interval increases. $\boldsymbol{F}$, Waterfall graph of RPTs in a HFD $24+16$ mouse, revealing, in contrast to controls $(\boldsymbol{E})$, axons require at least a $0.8 \mathrm{~ms}$ interval before they can conduct the second stimulus (red arrow). $\mathbf{G}$, Graph comparing RPT recovery in control (green) and HFD (red) $4+36$ mice, expressed as the recovery in amplitude of the second as a proportion of the first sCAP, is similar between the control and HFD $4+36$ mice. $\boldsymbol{H}$, Graph comparing RPT recovery in control (green) and HFD (red) $24+16$ mice, expressed as the recovery in amplitude of the second as a proportion of the first sCAP, is than larger-diameter myelinated axons (4$6 \mu \mathrm{m}$ ) in control mice (Fig. 7E). In $24+16$ HFD Tn-XXL mice, we found a small, but very significant, decrease in $\left[\mathrm{Ca}^{2+}\right]_{\mathrm{i}}$ in both myelinated and unmyelinated saphenous nerve axons compared with Tn-XXL controls (Fig. 7F; $p<0.0001$, unpaired $t$ test, $n=9$ animals per group). Taken as a whole, our results show that a 16 week HFD diet caused an unexpected decease in $\left[\mathrm{Ca}^{2+}\right]_{i}$ in vivo in both myelinated and unmyelinated saphenous nerve axons, compared with control mice on control diet.

\section{Discussion}

PN in prediabetes and T2D exhibits distalto-proximal nerve damage and axonal dysfunction; however, underlying molecular mechanisms are undefined. Our clinical studies indicate that dyslipidemia, through the metabolic syndrome, contributes to $\mathrm{PN}$, even in normoglycemic individuals (Callaghan et al., 2020). We also reported that long-chain SFAs impair mitochondrial MMP, bioenergetics capacity, ATP production, and motility in cultured DRG neurons (Rumora et al., 2018, 2019a). Herein, we postulated that similar mechanisms contribute to sensory neuron degeneration in vivo during PN. Using a HFD prediabetic mouse model that develops PN (O'Brien et al., 2014) and accumulates ectopic nerve fat (O'Brien et al., 2020), we characterized mitochondrial function within the saphenous nerve, a sensory nerve of bundled axons that relies on mitochondrial axonal trafficking to maintain energy homeostasis (Sajic et al., 2013). Surprisingly, HFD only marginally affected mitochondrial transport; however, it significantly increased stationary mitochondrial size, lowered MMP, and impaired saphenous nerve conduction at physiological frequencies. This suggests that lower MMP in HFD mitochondria may produce insufficient ATP to sustain physiological conduction. Additionally, HFD decreased $\left[\mathrm{Ca}^{2+}\right]_{\mathrm{i}}$ and increased DRG PGC1 $\alpha$ expression.

We first evaluated whether HFD feeding has a sex-specific effect on PN. All male and female HFD mice developed metabolic dysfunction and tactile hypersensitivity. Although HFD female mice had delayed onset of tactile hypersensitivity, both sexes exhibited similar tactile responses at 20 and 40 weeks when in vivo experiments were conducted. Therefore, the molecular changes underlying PN may not be sex-

\section{$\leftarrow$}

significantly prolonged in HFD versus control mice. ${ }^{* *} p<0.01$ (two-way ANOVA). $n=3$ control; $n=6$ HFD. This shows impaired ability of HFD axons to conduct closely spaced impulses. $* p<0.05$. 

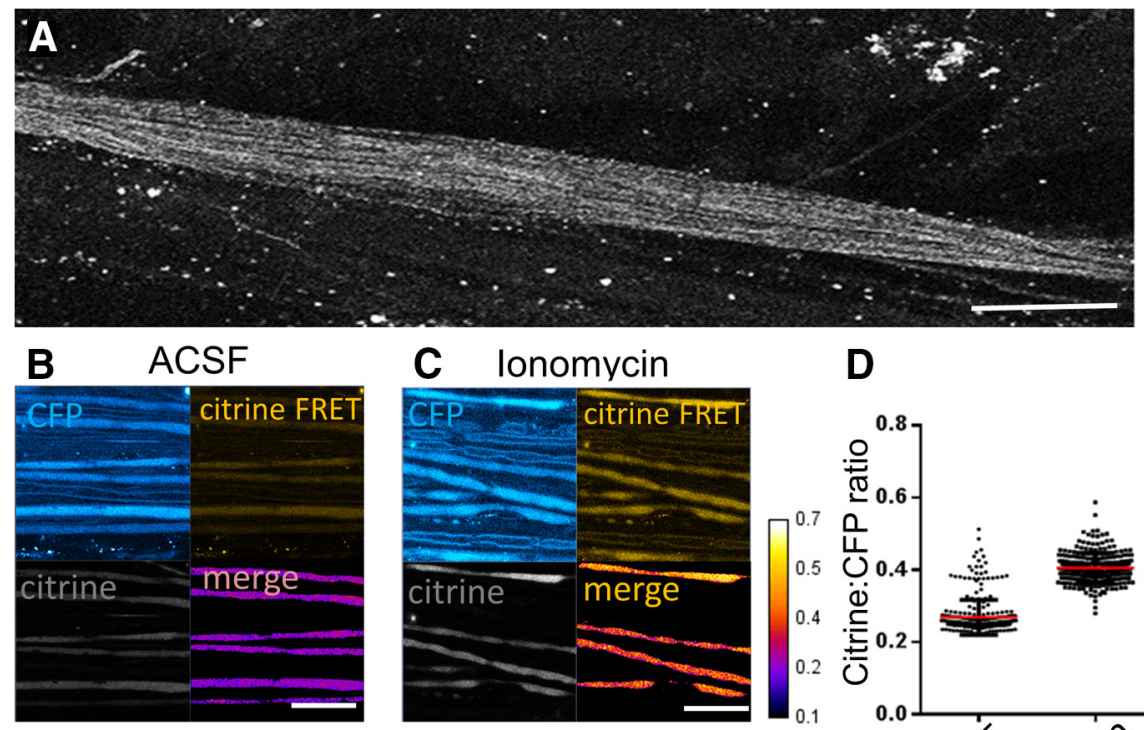

D

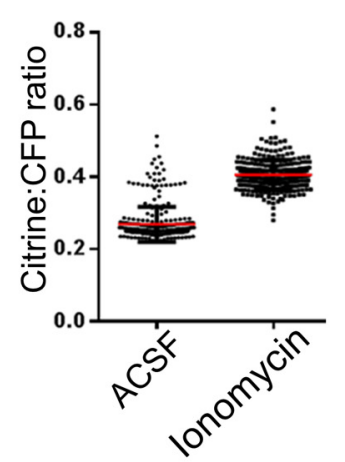

E

$9+5$ weeks diet
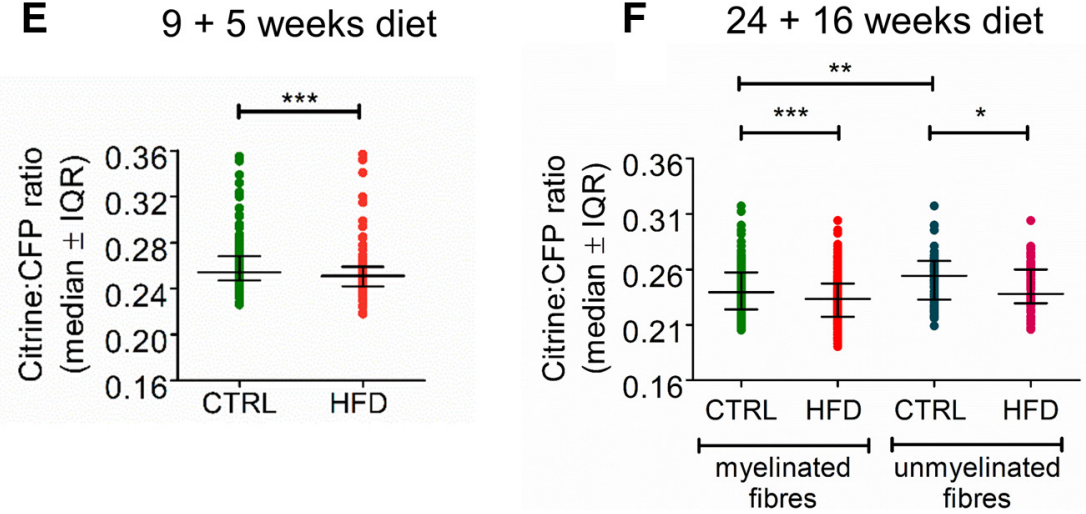

Figure 7. HFD mice exhibit reduced $\left[\mathrm{Ca}^{2+}\right]_{\mathrm{i}}$ levels in saphenous nerve axons in vivo. $\boldsymbol{A}$, Confocal image of an exposed saphenous nerve in vivo in a Tn-XXL mouse. Scale bar, $400 \mu \mathrm{m}$. $\boldsymbol{B}, \boldsymbol{C}$, High-magnification confocal image in CFP, citrine, and citrine FRET channels with a merge of CFP and citrine FRET, of saphenous nerve axons bathed in vivo with aCSF (B) or ionomycin $(100 \mu \mathrm{m})$ for $20 \mathrm{~min}(\boldsymbol{C})$. Scale bar, $50 \mu \mathrm{m}$. D, Citrine to (FP intensity ratio increases after incubating control Tn-XXL mouse saphenous nerve axons in ionomycin $(100 \mu \mathrm{m})$ for $20 \mathrm{~min}$ ( $n=4$ mice per group, $n=257-318$ axons per group). $\boldsymbol{E}$, Pilot data of HFD $9+5$ mice exhibit reduced $\left[\mathrm{Ca}^{2+}\right]_{\mathrm{i}}$ in myelinated axons compared with controls. ${ }^{*} p<0.05$ (Mann-Whitney test). Data are mean \pm SEM. $n=4$ control mice with $n=318$ axons; $n=4$ HFD mice with $n=148$ axons. $\boldsymbol{F}$, HFD $24+16$ mice exhibit reduced $\left[\mathrm{Ca}^{2+}\right]_{\mathrm{i}}$ in both myelinated and unmyelinated fibers compared with controls. ${ }^{* *} p<0.01$; ${ }^{* *} p<0.001$; Kruskal-Wallis test. Data are mean \pm SEM. $n=9$ control mice with $n=70$ axons; $n=10$ HFD mice with $n=60$ axons. Weight gain and impaired glucose tolerance in Tn-XXL (calcium reporter) mice are shown in Extended Data Figure 7-1.

dependent so we used HFD male mice throughout the study. Male HFD $4+36$ mice had elevated plasma cholesterol and phospholipids associated with PN, similar to previous studies (Tesfaye et al., 2005). Alterations in cholesterol level (Madamanchi and Runge, 2007) and phospholipid composition (Lu and Claypool, 2015) are reported to compromise mitochondrial function in dyslipidemia models, so we evaluated the effect of HFD on mitochondrial events in vivo.

We anticipated HFD-induced mitochondrial transport deficiencies in vivo in saphenous nerve based on our in vitro data (Rumora et al., 2018); however, no significant differences were observed. Indeed, short-term HFD increased motile mitochondria numbers in young mice, but not in old mice. The response to short-term HFD in young mice may represent a compensatory mechanism to counteract partial MMP loss and impaired energy production (Baqri et al., 2009), which may fail in old animals. In young mice, healthy polarized mitochondria are rapidly transported into areas of localized, experimentally induced saphenous nerve axon damage to avert local bioenergetics collapse (Sajic et al., 2018). The discrepancy between our in vitro and in vivo results perhaps reflects myelination state, which is absent in vitro but present around many saphenous axons in vivo (Ohno et al., 2011).

We found that HFD significantly increased the average mitochondrial size in vivo, indicating a shift toward mitochondrial biogenesis. Similar to PGC1 $\alpha$ overexpressing cardiac myocytes (Russell et al., 2004), we observed increased PGC1 $\alpha$ protein levels in HFD DRG that correlated with increases in mitochondrial size. Since PGC1 $\alpha$ stimulates fatty acid oxidation (Scarpulla, 2011; Cheng et al., 2018), higher PGC1 $\alpha$ levels in HFD DRGs may be a compensatory mechanism to boost mitochondrial biogenesis and offset MMP loss and energy deficits (Zhang et al., 2019). PGC1 $\alpha$ can also induce mitochondrial fusion by activating MFN2 (Zorzano, 2009). However, as seen in skeletal muscle (Jheng et al., 2012) and hepatic tissue (Lionetti et al., 2014), we found no change in DRG and sural MFN2 protein levels, suggesting that mitochondrial fusion is not responsible for the HFD-induced increases in axonal mitochondrial size in sensory nerves. PGC1 $\alpha$ also regulates mitochondrial metabolism and apoptosis through VDAC1/2 (Shoshan-Barmatz et al., 2010; Gill et al., 2019). Indeed, we found a significant decrease in VDAC1/2 protein expression in HFD sensory nerves. Therefore, increased DRG PGC1 $\alpha$ expression may not be sufficient to offset mitochondrial energy deficits in the sensory nerve axons resulting in a loss of VDAC1/2 expression indicative of mitochondrial dysfunction.

In vitro, SFAs decreased MMP in DRG neurons (Rumora et al., 2018, 2019a,b), and HFD exerted a similar effect in vivo, even after short-term HFD. Similarly, SFAs decreased MMP in C2C12 mouse muscle cells (Jheng et al., 2012) and primary mouse cardiomyocytes (Joseph et al., 2016). The partial MMP loss we measured in HFD mouse saphenous mitochondria did not completely abrogate axonal function. This suggests that elevated SFAs in the HFD reduce MMP by partially uncoupling the proton gradient from ATP production in the saphenous nerve. Partial uncoupling of the proton gradient allows more electron carriers $\left(\mathrm{NADH} / \mathrm{FADH}_{2}\right)$ to enter the electron transport chain, thereby increasing fatty acid $\beta$ oxidation and ATP production to sustain axonal function (Skulachev, 1998; Hinder et al., 2019). However, increased energy demand may result in a complete loss of MMP and energy production within HFD saphenous nerves. 
Therefore, at low energy demand, the HFD saphenous nerve conducted; however, increasing demand to $50 \mathrm{~Hz}$ simulation, levels commensurate with daily activities, doe instance, walking (Prochazka and Gorassini, 1998), prompted limited conduction, suggesting inability to supply additional ATP for impulse conduction. This progressive conduction slowing/blocking likewise precedes overt PN in diabetics (Dyck et al., 2005). Moreover, further MMP reductions may completely depolarize axonal mitochondria, triggering degradation, and chronic ATP shortages might increase intra-axonal $\mathrm{Na}^{+}$levels, causing axonal degeneration (Stys et al., 1992). Conversely, healthy saphenous axons from control mice readily conducted at $50 \mathrm{~Hz}$, as expected since MMP drives ATP synthesis (Nicholls and Ferguson, 2013). Indeed, impulse conduction in healthy saphenous nerve coincides with increased ATP production (van Hameren et al., 2019). Our study is the first, to our knowledge, to quantify MMP changes in adult mammalian axons during impulse conduction in vivo. The partial dissipation of MMP may be an important step to increase ATP synthesis during impulse conduction.

Saphenous nerve MMP and axonal conduction were impaired, although distal axonal terminals were structurally intact in the hairy skin they innervate on the medial foot dorsum, supporting human studies demonstrating conduction defects before PN onset (Dyck et al., 2005). HFD animals developed PN in glabrous skin on the plantar foot innervated by the sciatic nerve, evidenced by lower IENFD compared with controls. This result is supported by studies evaluating effects of 45\%-60\% HFD in prediabetic mice on glabrous skin, which exhibit IENFD loss as a PN marker (Vincent et al., 2009; Rumora et al., 2019b; O'Brien et al., 2020). The differential impact of HFD on IENFD in glabrous versus hairy skin may reflect effects of increased body weight on the plantar foot in HFD mice (Collongues et al., 2018).

Importantly, we found lower saphenous axonal $\left[\mathrm{Ca}^{2+}\right]_{i}$ in HFD mice versus controls before overt PN. SFAs increase $\left[\mathrm{Ca}^{2+}\right]_{\mathrm{i}}$ at the expense of endoplasmic reticulum $\mathrm{Ca}^{2+}$ stores, prompting excessive intramitochondrial $\mathrm{Ca}^{2+}$ in T2D models (Ly et al., 2017). Palmitate raises $\left[\mathrm{Ca}^{2+}\right]_{\mathrm{i}}$ in mouse (Remizov et al., 2003) and human (Gwiazda et al., 2009) primary $\beta$ cells, rat INS-1 insulinoma cells (Schnell et al., 2007), and mouse podocytes (Xu et al., 2015), and elevated $\left[\mathrm{Ca}^{2+}\right]_{\mathrm{i}}$ is a unifying axonal degeneration mechanism across multiple neuronal injury types (LoPachin and Lehning, 1997), including diabetic PN (Fernyhough and Calcutt, 2010). DRGs from streptozotocininduced Type 1 diabetes and genetic leptin signaling-deficient $d b / d b$ T2D mice exhibit elevated $\left[\mathrm{Ca}^{2+}\right]_{\mathrm{i}}$ (Kostyuk et al., 1999); however, shorter diabetes durations induced no discernible effects (Kostyuk et al., 2001), suggesting that calcium dyshomeostasis occurs early. Thus, lower $\left[\mathrm{Ca}^{2+}\right]_{\mathrm{i}}$ may be an earlier pathologic consequence of metabolic disturbance, and higher $\left[\mathrm{Ca}^{2+}\right]_{\mathrm{i}}$ likely occurs during later irreversible stages of PN.

Our study had several limitations. First, although we did not find sex differences in the development of metabolic dysfunction or tactile hypersensitivity, there may be sex dimorphism in mitochondrial activity in vivo in response to the HFD. Future studies will assess the effect of HFD on MMP, $\left[\mathrm{Ca}^{2+}\right]_{i}$, and PGC1 $\alpha$ expression in the saphenous nerve from female and male mice. Second, we identified an early decrease in axonal $\left[\mathrm{Ca}^{2+}\right]_{\mathrm{i}}$ before overt PN in HFD mice, suggesting that decreased axonal $\left[\mathrm{Ca}^{2+}\right]_{\mathrm{i}}$ is an early pathologic response to metabolic dysfunction and that axonal $\left[\mathrm{Ca}^{2+}\right]_{\mathrm{i}}$ may increase in later-stage $\mathrm{PN}$ pathogenesis. Although we did not test this hypothesis herein, future studies will focus on calcium flux in saphenous nerve axons. Although a low number of biological replicates is a study limitation, we observed replicable and significant results. Future studies will focus on increasing the number of mice for sCAP analyses and axonal $\left[\mathrm{Ca}^{2+}\right]_{\mathrm{i}}$ assessment, to reduce variance between animals.

Our findings lend new insight into early pathologic events in saphenous nerve axons underlying PN development during HFD-induced prediabetes. MMP reduction was a key pathogenic event and may consequently reduce the ability of mitochondria to increase ATP production to meet energy demands, which is relevant to daily activities, such as sensory perception during walking. Impairment of mitochondrial function was also associated with several compensatory mechanisms, including increased mitochondrial size and transport and elevated PGC1 $\alpha$ expression. Importantly, these mechanisms were accompanied by reduced $\left[\mathrm{Ca}^{2+}\right]_{\mathrm{i}}$, which may have profound consequences for axonal signaling, maintenance, and regeneration. We suggest that diminished MMP in HFD mitochondria creates unbalanced axonal energy supply and demand concomitant with decreased axonal $\left[\mathrm{Ca}^{2+}\right]_{\mathrm{i}}$, thereby contributing to $\mathrm{PN}$ progression in prediabetes.

\section{References}

Andrews S, Gilley J, Coleman MP (2010) Difference Tracker: ImageJ plugins for fully automated analysis of multiple axonal transport parameters. J Neurosci Methods 193:281-287.

Baqri RM, Turner BA, Rheuben MB, Hammond BD, Kaguni LS, Miller KE (2009) Disruption of mitochondrial DNA replication in Drosophila increases mitochondrial fast axonal transport in vivo. PLoS One 4:e7874.

Braune HJ (1999) Testing of the refractory period in sensory nerve fibres is the most sensitive method to assess beginning polyneuropathy in diabetics. Electromyogr Clin Neurophysiol 39:355-359.

Callaghan BC, Little AA, Feldman EL, Hughes RA (2012) Enhanced glucose control for preventing and treating diabetic neuropathy. Cochrane Database Syst Rev 6:CD007543.

Callaghan BC, Xia R, Reynolds E, Banerjee M, Rothberg AE, Burant CF, Villegas-Umana E, Pop-Busui R, Feldman EL (2016a) Association between metabolic syndrome components and polyneuropathy in an obese population. JAMA Neurol 73:1468-1476.

Callaghan BC, Xia R, Banerjee M, de Rekeneire N, Harris TB, Newman AB, Satterfield S, Schwartz AV, Vinik AI, Feldman EL, Strotmeyer ES, Health ABC Study (2016b) Metabolic syndrome components are associated with symptomatic polyneuropathy independent of glycemic status. Diabetes Care 39:801-807.

Callaghan BC, Gao L, Li Y, Zhou X, Reynolds E, Banerjee M, Pop-Busui R, Feldman EL, Ji L (2018) Diabetes and obesity are the main metabolic drivers of peripheral neuropathy. Ann Clin Transl Neurol 5:397-405.

Callaghan BC, Reynolds EL, Banerjee M, Chant E, Villegas-Umana E, Gardner TW, Votruba K, Giordani B, Pop-Busui R, Pennathur S, Feldman EL (2020) The prevalence and determinants of cognitive deficits and traditional diabetic complications in the severely obese. Diabetes Care 43:683-690.

Chada SR, Hollenbeck PJ (2004) Nerve growth factor signaling regulates motility and docking of axonal mitochondria. Curr Biol 14:1272-1276.

Chen M, Wang Y, Qu A (2010) PGC-1 alpha accelerates cytosolic $\mathrm{Ca}^{2+}$ clearance without disturbing $\mathrm{Ca}^{2+}$ homeostasis in cardiac myocytes. Biochem Biophys Res Commun 396:894-900.

Cheng CF, Ku HC, Lin H (2018) PGC-1 $\alpha$ as a pivotal factor in lipid and metabolic regulation. Int J Mol Sci 19:3447.

Collongues N, Samama B, Schmidt-Mutter C, Chamard-Witkowski L, Debouverie M, Chanson JB, Antal MC, Benardais K, de Seze J, Velten M, Boehm N (2018) Quantitative and qualitative normative dataset for intraepidermal nerve fibers using skin biopsy. PLoS One 13:e0191614.

Dyck PJ, O’Brien PC, Litchy WJ, Harper CM, Klein CJ, Dyck BD (2005) Monotonicity of nerve tests in diabetes: subclinical nerve dysfunction precedes diagnosis of polyneuropathy. Diabetes Care 28:2192-2200.

Eid S, Sas KM, Abcouwer SF, Feldman EL, Gardner TW, Pennathur S, Fort PE (2019) New insights into the mechanisms of diabetic complications: role of lipids and lipid metabolism. Diabetologia 62:1539-1549. 
Feldman EL, Callaghan BC, Pop-Busui R, Zochodne DW, Wright DE, Bennett DL, Bril V, Russell JW, Viswanathan V (2019) Diabetic neuropathy. Nat Rev Dis Primers 5:41.

Fernyhough P, Calcutt NA (2010) Abnormal calcium homeostasis in peripheral neuropathies. Cell Calcium 47:130-139.

Gill JF, Delezie J, Santos G, McGuirk S, Schnyder S, Frank S, Rausch M, StPierre J, Handschin C (2019) Peroxisome proliferator-activated receptor $\gamma$ coactivator $1 \alpha$ regulates mitochondrial calcium homeostasis, sarcoplasmic reticulum stress, and cell death to mitigate skeletal muscle aging. Aging Cell 18:e12993.

Gwiazda KS, Yang TL, Lin Y, Johnson JD (2009) Effects of palmitate on ER and cytosolic $\mathrm{Ca}^{2+}$ homeostasis in beta cells. Am J Physiol Endocrinol Metab 296:E690-E701.

Hinder LM, Sas KM, O’Brien PD, Backus C, Kayampilly P, Hayes JM, Lin CM, Zhang H, Shanmugam S, Rumora AE, Abcouwer SF, Brosius FC, Pennathur S, Feldman EL (2019) Mitochondrial uncoupling has no effect on microvascular complications in type 2 diabetes. Sci Rep 9:881.

Jheng HF, Tsai PJ, Guo SM, Kuo LH, Chang CS, Su IJ, Chang CR, Tsai YS (2012) Mitochondrial fission contributes to mitochondrial dysfunction and insulin resistance in skeletal muscle. Mol Cell Biol 32:309-319.

Joseph LC, Barca E, Subramanyam P, Komrowski M, Pajvani U, Colecraft HM, Hirano M, Morrow JP (2016) Inhibition of NAPDH oxidase 2 (NOX2) prevents oxidative stress and mitochondrial abnormalities caused by saturated fat in cardiomyocytes. PLoS One 11:e0145750.

Kostyuk E, Svichar N, Shishkin V, Kostyuk P (1999) Role of mitochondrial dysfunction in calcium signalling alterations in dorsal root ganglion neurons of mice with experimentally-induced diabetes. Neuroscience 90:535-541.

Kostyuk E, Voitenko N, Kruglikov I, Shmigol A, Shishkin V, Efimov A, Kostyuk P (2001) Diabetes-induced changes in calcium homeostasis and the effects of calcium channel blockers in rat and mice nociceptive neurons. Diabetologia 44:1302-1309.

Lionetti L, Mollica MP, Donizzetti I, Gifuni G, Sica R, Pignalosa A, Cavaliere G, Gaita M, De Filippo C, Zorzano A, Putti R (2014) High-lard and highfish-oil diets differ in their effects on function and dynamic behaviour of rat hepatic mitochondria. PLoS One 9:e92753.

LoPachin RM, Lehning EJ (1997) Mechanism of calcium entry during axon injury and degeneration. Toxicol Appl Pharmacol 143:233-244.

Lu YW, Claypool SM (2015) Disorders of phospholipid metabolism: an emerging class of mitochondrial disease due to defects in nuclear genes. Front Genet 6:3.

Ly LD, Xu S, Choi SK, Ha CM, Thoudam T, Cha SK, Wiederkehr A, Wollheim CB, Lee IK, Park KS (2017) Oxidative stress and calcium dysregulation by palmitate in type 2 diabetes. Exp Mol Med 49:e291.

Madamanchi NR, Runge MS (2007) Mitochondrial dysfunction in atherosclerosis. Circ Res 100:460-473.

Mank M, Santos AF, Direnberger S, Mrsic-Flogel TD, Hofer SB, Stein V, Hendel T, Reiff DF, Levelt C, Borst A, Bonhoeffer T, Hübener M, Griesbeck O (2008) A genetically encoded calcium indicator for chronic in vivo two-photon imaging. Nat Methods 5:805-811.

Morris RL, Hollenbeck PJ (1993) The regulation of bidirectional mitochondrial transport is coordinated with axonal outgrowth. J Cell Sci 104: 917927.

Nicholls DG, Ferguson SJ (2013) Respiratory chains. In: Bioenergetics, Ed 4 (Nicholls DG, Ferguson SJ, eds), pp 91-95. Boston: Academic.

O’Brien PD, Sakowski SA, Feldman EL (2014) Mouse models of diabetic neuropathy. ILAR J 54:259-272.

O’Brien PD, Guo K, Eid SA, Rumora AE, Hinder LM, Hayes JM, Mendelson FE, Hur J, Feldman EL (2020) Integrated lipidomic and transcriptomic analyses identify altered nerve triglycerides in mouse models of prediabetes and type 2 diabetes. Dis Model Mech 13:dmm042101.

Obrosova IG, Ilnytska O, Lyzogubov VV, Pavlov IA, Mashtalir N, Nadler JL, Drel VR (2007) High-fat diet induced neuropathy of pre-diabetes and obesity: effects of "healthy" diet and aldose reductase inhibition. Diabetes 56:2598-2608.

Ohno N, Kidd GJ, Mahad D, Kiryu-Seo S, Avishai A, Komuro H, Trapp BD (2011) Myelination and axonal electrical activity modulate the distribution and motility of mitochondria at CNS nodes of Ranvier. J Neurosci 31:7249-7258
Prochazka A, Gorassini M (1998) Models of ensemble firing of muscle spindle afferents recorded during normal locomotion in cats. J Physiol 507:277-291.

Remizov O, Jakubov R, Düfer M, Krippeit Drews P, Drews G, Waring M, Brabant G, Wienbergen A, Rustenbeck I, Schöfl C (2003) Palmitateinduced $\mathrm{Ca}^{2+}$-signaling in pancreatic beta cells. Mol Cell Endocrinol 212:1-9.

Rizzuto R, De Stefani D, Raffaello A, Mammucari C (2012) Mitochondria as sensors and regulators of calcium signalling. Nat Rev Mol Cell Biol 13:566-578.

Rumora AE, Lentz SI, Hinder LM, Jackson SW, Valesano A, Levinson GE, Feldman EL (2018) Dyslipidemia impairs mitochondrial trafficking and function in sensory neurons. FASEB J 32:195-207.

Rumora AE, LoGrasso G, Haidar JA, Dolkowski JJ, Lentz SI, Feldman EL (2019a) Chain length of saturated fatty acids regulates mitochondrial trafficking and function in sensory neurons. J Lipid Res 60:58-70.

Rumora AE, LoGrasso G, Hayes JM, Mendelson FE, Tabbey MA, Haidar JA, Lentz SI, Feldman EL (2019b) The divergent roles of dietary saturated and monounsaturated fatty acids on nerve function in murine models of obesity. J Neurosci 39:3770-3781.

Russell LK, Mansfield CM, Lehman JJ, Kovacs A, Courtois M, Saffitz JE, Medeiros DM, Valencik ML, McDonald JA, Kelly DP (2004) Cardiacspecific induction of the transcriptional coactivator peroxisome proliferator-activated receptor gamma coactivator-1alpha promotes mitochondrial biogenesis and reversible cardiomyopathy in a developmental stagedependent manner. Circ Res 94:525-533.

Saeedi P, Petersohn I, Salpea P, Malanda B, Karuranga S, Unwin N, Colagiuri S, Guariguata L, Motala AA, Ogurtsova K, Shaw JE, Bright D, Williams R, IDF Diabetes Atlas Committee (2019) Global and regional diabetes prevalence estimates for 2019 and projections for 2030 and 2045: results from the International Diabetes Federation Diabetes Atlas, $9^{\text {th }}$ edition. Diabetes Res Clin Pract 157:107843.

Sajic M (2014) Mitochondrial dynamics in peripheral neuropathies. Antioxid Redox Signal 21:601-620.

Sajic M, Mastrolia V, Lee CY, Trigo D, Sadeghian M, Mosley AJ, Gregson NA, Duchen MR, Smith KJ (2013) Impulse conduction increases mitochondrial transport in adult mammalian peripheral nerves in vivo. PLoS Biol 11:e1001754.

Sajic M, Ida KK, Canning R, Gregson NA, Duchen MR, Smith KJ (2018) Mitochondrial damage and "plugging" of transport selectively in myelinated, small-diameter axons are major early events in peripheral neuroinflammation. J Neuroinflammation 15:61

Savelieff MG, Callaghan BC, Feldman EL (2020) The emerging role of dyslipidemia in diabetic microvascular complications. Curr Opin Endocrinol Diabetes Obes 27:115-123.

Scarpulla RC (2011) Metabolic control of mitochondrial biogenesis through the PGC-1 family regulatory network. Biochim Biophys Acta 1813:12691278.

Schnell S, Schaefer M, Schofl C (2007) Free fatty acids increase cytosolic free calcium and stimulate insulin secretion from beta cells through activation of GPR40. Mol Cell Endocrinol 263:173-180.

Schonfeld P, Wojtczak L (2016) Short- and medium-chain fatty acids in energy metabolism: the cellular perspective. J Lipid Res 57:943-954.

Shoshan-Barmatz V, De Pinto V, Zweckstetter M, Raviv Z, Keinan N, Arbel $\mathrm{N}$ (2010) VDAC, a multi-functional mitochondrial protein regulating cell life and death. Mol Aspects Med 31:227-285.

Skulachev VP (1998) Uncoupling: new approaches to an old problem of bioenergetics. Biochim Biophys Acta 1363:100-124.

Smith KJ (1980) A sensitive method for the detection and quantification of conduction deficits in nerve. J Neurol Sci 48:191-199.

Stys PK, Waxman SG, Ransom BR (1992) Ionic mechanisms of anoxic injury in mammalian CNS white matter: role of $\mathrm{Na}^{+}$channels and $\mathrm{Na}(+)-\mathrm{Ca}^{2+}$ exchanger. J Neurosci 12:430-439.

Tesfaye S, Chaturvedi N, Eaton SE, Ward JD, Manes C, Ionescu-Tirgoviste C, Witte DR, Fuller JH, EURODIAB Prospective Complications Study Group (2005) Vascular risk factors and diabetic neuropathy. N Engl J Med 352:341-350.

van Hameren G, Campbell G, Deck M, Berthelot J, Gautier B, Quintana P, Chrast R, Tricaud N (2019) In vivo real-time dynamics of ATP and ROS production in axonal mitochondria show decoupling in mouse models of peripheral neuropathies. Acta Neuropathol Commun 7:86. 
Vincent AM, Hayes JM, McLean LL, Vivekanandan-Giri A, Pennathur S, Feldman EL (2009) Dyslipidemia-induced neuropathy in mice: the role of oxLDL/LOX-1. Diabetes 58:2376-2385.

Vincent AM, Edwards JL, McLean LL, Hong Y, Cerri F, Lopez I, Quattrini A, Feldman EL (2010) Mitochondrial biogenesis and fission in axons in cell culture and animal models of diabetic neuropathy. Acta Neuropathol 120:477-489.

Xu S, Nam SM, Kim JH, Das R, Choi SK, Nguyen TT, Quan X, Choi SJ, Chung CH, Lee EY, Lee IK, Wiederkehr A, Wollheim CB, Cha SK, Park KS (2015) Palmitate induces ER calcium depletion and apoptosis in mouse podocytes subsequent to mitochondrial oxidative stress. Cell Death Dis 6:e1976.
Zhang CL, Ho PL, Kintner DB, Sun D, Chiu SY (2010) Activity-dependent regulation of mitochondrial motility by calcium and $\mathrm{Na}$ / K-ATPase at nodes of Ranvier of myelinated nerves. J Neurosci 30:3555-3566.

Zhang Q, Lei YH, Zhou JP, Hou YY, Wan Z, Wang HL, Meng H (2019) Role of PGC- $1 \alpha$ in mitochondrial quality control in neurodegenerative diseases. Neurochem Res 44:2031-2043.

Zorova LD, Popkov VA, Plotnikov EY, Silachev DN, Pevzner IB, Jankauskas SS, Babenko VA, Zorov SD, Balakireva AV, Juhaszova M, Sollott SJ, Zorov DB (2018) Mitochondrial membrane potential. Anal Biochem 552:50-59.

Zorzano A (2009) Regulation of mitofusin-2 expression in skeletal muscle. Appl Physiol Nutr Metab 34:433-439. 